\title{
Feynman rules for the rational part of the electroweak 1-loop amplitudes
}

\author{
M.V. Garzelli, ${ }^{a, b}$ I. Malamos ${ }^{c}$ and R. Pittau ${ }^{a}$ \\ a Departamento de Física Teórica y del Cosmos y CAFPE, Universidad de Granada, \\ E-18071 Granada, Spain \\ a INFN Milano, \\ I-20133 Milano, Italy \\ ${ }^{c}$ Department of Theoretical High Energy Physics, Institute for Mathematics, \\ Astrophysics and Particle Physics, Radboud Universiteit Nijmegen, \\ 6525 AJ Nijmegen, the Netherlands \\ E-mail: garzelli@to.infn.it, J.Malamos@science.ru.nl, pittau@ugr.es
}

AbStRaCT: We present the complete set of Feynman rules producing the rational terms of kind $\mathrm{R}_{2}$ needed to perform any 1-loop calculation in the Electroweak Standard Model. Our results are given both in the 't Hooft-Veltman and in the Four Dimensional Helicity regularization schemes. We also verified, by using both the 't Hooft-Feynman gauge and the Background Field Method, a huge set of Ward identities -up to 4-points- for the complete rational part of the Electroweak amplitudes. This provides a stringent check of our results and, as a by-product, an explicit test of the gauge invariance of the Four Dimensional Helicity regularization scheme in the complete Standard Model at 1-loop. The formulae presented in this paper provide the last missing piece for completely automatizing, in the framework of the OPP method, and in any other approach using 4-dimensional numerators, the 1-loop calculations in the $\mathrm{SU}(3) \times \mathrm{SU}(2) \times \mathrm{U}(1)$ Standard Model.

KEYwords: NLO Computations, Standard Model 


\section{Contents}

1 Introduction $\quad 2$

2 Theory, facts and conjectures on $R, R_{1}$ and $R_{2}$

3 Results $\quad 5$

$\begin{array}{lll}3.1 & \text { Electroweak effective vertices with } 2 \text { external legs } & 6\end{array}$

3.1.1 Scalar-Scalar effective vertices 6

$\begin{array}{lll}3.1 .2 & \text { Vector-Vector effective vertices } & 7\end{array}$

$\begin{array}{lll}\text { 3.1.3 Fermion-Fermion effective vertices } & 7\end{array}$

$\begin{array}{lll}3.2 & \text { Electroweak effective vertices with } 3 \text { external legs } & 8\end{array}$

3.2.1 Scalar-Fermion-Fermion effective vertices 8

$\begin{array}{ll}3.2 .2 \text { Vector-Fermion-Fermion effective vertices } & 10\end{array}$

$\begin{array}{lll}3.2 .3 & \text { Scalar-Scalar-Scalar effective vertices } & 12\end{array}$

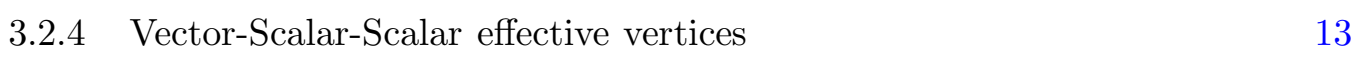

$\begin{array}{lll}3.2 .5 & \text { Scalar-Vector-Vector effective vertices } & 14\end{array}$

$\begin{array}{ll}3.2 .6 & \text { Vector-Vector-Vector effective vertices } \\ \end{array}$

$\begin{array}{lll}3.3 & \text { Electroweak effective vertices with } 4 \text { external legs } & 15\end{array}$

$\begin{array}{lll}\text { 3.3.1 Scalar-Scalar-Scalar-Scalar effective vertices } & 16\end{array}$

$\begin{array}{ll}\text { 3.3.2 Vector-Vector-Vector-Vector effective vertices } & 16\end{array}$

$\begin{array}{lll}\text { 3.3.3 Scalar-Scalar-Vector-Vector effective vertices } & 19\end{array}$

$\begin{array}{lll}3.4 & \text { Mixed Electroweak/QCD corrections } & 21\end{array}$

3.4.1 Gluon-Quark-Quark effective vertex 21

4 Tests and findings $\quad 22$

5 Conclusions $\quad 22$

$\begin{array}{ll}\text { A Ward identities } & \mathbf{2 3}\end{array}$

A.1 Ward identities involving VV, VS and SS 24

A.2 Ward identities involving VFF, SFF and FF 24

A.3 Ward identities involving VVV, VVS and VV 24

A.4 Ward identities involving VVS, VSS and VS 25

A.5 Ward identities involving VSS, SSS and SS 26

A.6 Ward identities involving VVVV, VVVS and VVV 27

A.7 Ward identities involving SSSS, VSSS and SSS 28 


\section{Introduction}

The complete automation of the 1-loop calculations for LHC and ILC physics is nowadays a feasible task [1-5]. The advent of the OPP reduction method [6], together with the concept of multiple cuts [7], allowed to revitalize the Unitarity Techniques [8-10], by reducing the computation of 1-loop amplitudes to a problem with the same conceptual complexity of a tree level calculation, resulting in achievements that were inconceivable only a few years ago [11].

The main idea of the OPP based techniques is directly extracting, from the 1-loop amplitude, the coefficients of the (known) scalar loop functions. This task can be reached in a completely numerical way by opening the loop and transforming the 1-loop amplitude in a tree level object with 2 more legs, that can be calculated, at the integrand level, by using the same recursion relations $[12,13]$ that allow a very efficient computation of complicated multi-leg tree level processes $[14,15]$. A second possible option is that one of the so-called Generalized Unitarity methods $[16,17]$, where tree-level amplitudes are glued together.

Both procedures only allow the extraction of the Cut Constructible (CC) part of the amplitude in 4 dimensions, while a left over piece, the rational part $\mathrm{R}$, needs to be derived separately. In the Generalized Unitarity approaches, that is achieved by computing the amplitude in different numbers of space-time dimensions, or via bootstrapping techniques [19-22], while, in the OPP approach, and in any other method using 4-dimensional numerators [23], $R$ is split in 2 pieces $R=R_{1}+R_{2}$. The first piece, $R_{1}$, is derivable in the same framework used to reconstruct the CC part of the amplitude, while $\mathrm{R}_{2}$ is computable through a special set Feynman rules for the theory at hand [24], to be used in a tree level-like computation.

Such a set of $R_{2}$ Feynman rules has been already derived for QED in [24] and for QCD in [25], and it is the main aim of the present paper to present the complete set of the $\mathrm{R}_{2}$ Feynman rules for the Standard Model (SM) of the Electroweak (EW) interactions. In addition, as a by-product, we use the derived formulae to explicitly check the gauge invariance of the Four Dimensional Helicity regularization scheme in the EW sector at 1loop, the motivation being that this is a very well studied subject in QCD [26-28], but, in our knowledge, very little can be found in the literature for the full EW Standard Model.

The outline of the paper is as follows. In section 2 we remind some facts on the origin of $\mathrm{R}$ and on the splitting $\mathrm{R}=\mathrm{R}_{1}+\mathrm{R}_{2}$. Section 3 contains the complete list of all possible special $R_{2}$ EW SM vertices in the 't Hooft-Feynman gauge and, in section 4, we describe the tests we performed on our formulae and our findings. Finally, our conclusions are drawn in section 5 and, in the appendix, we collect a list of Ward identities.

\section{Theory, facts and conjectures on $R, R_{1}$ and $R_{2}$}

Before carrying out our program, we spend a few words on the origin of R. Our starting point is the general expression for the integrand of a generic $m$-point one-loop 
(sub-) amplitude

$$
\bar{A}(\bar{q})=\frac{\bar{N}(\bar{q})}{\bar{D}_{0} \bar{D}_{1} \cdots \bar{D}_{m-1}}, \quad \bar{D}_{i}=\left(\bar{q}+p_{i}\right)^{2}-m_{i}^{2},
$$

where $\bar{q}$ is the integration momentum and where dimensional regularization is assumed, so that a bar denotes objects living in $n=4+\epsilon$ dimensions and a tilde represents $\epsilon$ dimensional quantities. When a $n$-dimensional index is contracted with a 4 -dimensional vector $v_{\mu}$, the 4 -dimensional part is automatically selected. For example

$$
\bar{q} \cdot v \equiv(q+\tilde{q}) \cdot v=q \cdot v, \quad \not{\psi} \equiv \bar{\gamma}_{\bar{\mu}} v^{\mu}=\psi \quad \text { and } \quad \bar{q}^{2}=q^{2}+\tilde{q}^{2} .
$$

The numerator function $\bar{N}(\bar{q})$ can be split into a 4-dimensional plus an $\epsilon$-dimensional part

$$
\bar{N}(\bar{q})=N(q)+\tilde{N}\left(\tilde{q}^{2}, q, \epsilon\right) .
$$

$N(q)$ lives in 4 dimensions, and can be therefore expanded in terms of 4dimensional denominators

$$
D_{i}=\left(q+p_{i}\right)^{2}-m_{i}^{2}=\bar{D}_{i}-\tilde{q}^{2} .
$$

Some among the coefficients in this expansion are interpreted, in the OPP method, as the desired coefficients of the 1-loop scalar integrals and can be determined numerically, while the mismatch between this expansion in terms of 4-dimensional denominators, and the $n$-dimensional denominators appearing in eq. (2.1), is the origin of the rational terms $R_{1}$. There exist at least two ways $[29,30]$ to compute $\mathrm{R}_{1}$, which allow to determine it by means of a purely numerical knowledge of the 4-dimensional CC part of the amplitude, while this does not seem to be possible for $\mathrm{R}_{2}$, whose origin is the term $\tilde{N}\left(\tilde{q}^{2}, q, \epsilon\right)$ in eq. (2.3), after integration over the loop momentum:

$$
\mathrm{R}_{2} \equiv \frac{1}{(2 \pi)^{4}} \int d^{n} \bar{q} \frac{\tilde{N}\left(\tilde{q}^{2}, q, \epsilon\right)}{\bar{D}_{0} \bar{D}_{1} \cdots \bar{D}_{m-1}} .
$$

However, $\mathrm{R}_{2}$ can be computed by extracting $\tilde{N}\left(\tilde{q}^{2}, q, \epsilon\right)$ from any given integrand $\bar{A}(\bar{q})$, which can be achieved by splitting, in the analytic expression of the numerator function, the $n$-dimensional integration momentum $\bar{q}$, the $n$-dimensional gamma matrices $\bar{\gamma}_{\bar{\mu}}$ and the $n$-dimensional metric tensor $\bar{g}^{\bar{\mu} \bar{\nu}}$ into a 4-dimensional component plus remaining pieces:

$$
\begin{aligned}
\bar{q} & =q+\tilde{q}, \\
\bar{\gamma}_{\bar{\mu}} & =\gamma_{\mu}+\tilde{\gamma}_{\tilde{\mu}}, \\
\bar{g}^{\bar{\mu} \bar{\nu}} & =g^{\mu \nu}+\tilde{g}^{\tilde{\mu} \tilde{\nu}} .
\end{aligned}
$$

Therefore, a practical way to determine $\mathrm{R}_{2}$ is computing analytically, by means of Feynman diagrams, once for all and with the help of eq. (2.6), tree-level like Feynman rules, namely effective vertices, by calculating the $\mathrm{R}_{2}$ part coming from all possible one-particle irreducible Green functions of the theory at hand, up to four external legs. The fact that four external legs are enough to account for $R_{2}$ is guaranteed by the ultraviolet nature 
of the rational terms, proved in $[31,32]$. This property does not hold, instead, for $R_{1}$, that, diagram by diagram, can give non vanishing contributions to any one-particle irreducible $m$-point function, because, even when finite, the tensor integrals generating $R_{1}$ are eventually expressed, via tensor reduction, in terms of linear combinations of 1-loop scalar functions that can be ultraviolet divergent. This fact prevents the possibility of calculating a finite set of effective vertices reproducing $R_{1}$.

Eq. (2.5) generates a set of simple basic integrals with up to 4 denominators, containing powers of $\tilde{q}$ and $\epsilon$ in the numerator. A list that exhausts all possibilities in the $\xi=1$ 't Hooft-Feynman gauge can be found in [25]. Notice, however, that, according to the chosen regularization scheme, results may differ. In eq. (2.5) we assume the 't Hooft-Veltman (HV) scheme, while in the Four Dimensional Helicity scheme (FDH), any explicit $\epsilon$ dependence in the numerator function is discarded before integration, such that

$$
\left.\mathrm{R}_{2}\right|_{F D H}=\frac{1}{(2 \pi)^{4}} \int d^{n} \bar{q} \frac{\tilde{N}\left(\tilde{q}^{2}, q, \epsilon=0\right)}{\bar{D}_{0} \bar{D}_{1} \cdots \bar{D}_{m-1}} .
$$

The asymmetric role played by $\mathrm{R}_{1}$ and $\mathrm{R}_{2}$ is somewhat annoying. As we have seen, $\mathrm{R}_{1}$ is directly connected with the (4-dimensional) $\mathrm{CC}$ part of the amplitude, and can be computed, even numerically, without any reference to Feynman diagrams, while $\mathrm{R}_{2}$ requires an analytic determination in terms of Feynman diagrams, so that one would like to be able to put both pieces on the same footing. Unfortunately, no easy direct connection between $\mathrm{R}_{2}$ and the CC part of the amplitude has been found so far (at least within our treatment at the integrand level) and, in the rest of this paragraph, we speculate a bit on this subject.

Reconstructing $\mathrm{R}_{2}$ numerically would require to detect "signs" of it in the $\mathrm{CC}$ part. For example, one could naively think that, by looking at any $q^{2}$ in the CC part, the $\tilde{q}^{2}$ dependence could be inferred via the replacement

$$
q^{2} \rightarrow q^{2}+\tilde{q}^{2}
$$

However, such a dependence is impossible to reconstruct numerically, when remaining in 4 dimensions, as it can be illustrated by considering the following simple 3 -point subamplitude:

$$
\mathrm{A} \equiv \frac{1}{(2 \pi)^{4}} \int d^{n} \bar{q} \frac{\left(q \cdot \ell_{3}\right)\left(q \cdot \ell_{4}\right)}{\bar{D}_{0} \bar{D}_{1} \bar{D}_{2}}
$$

where

$$
\left.\left.\ell_{3}^{\mu}=<\ell_{1}\left|\gamma^{\mu}\right| \ell_{2}\right], \quad \ell_{4}^{\mu}=<\ell_{2}\left|\gamma^{\mu}\right| \ell_{1}\right] \quad \text { with } \ell_{1,2}^{2}=0 .
$$

From the one hand, the 4-dimensional numerator $\left(q \cdot \ell_{3}\right)\left(q \cdot \ell_{4}\right)$ in eq. $(2.9)$ does not contain any $q^{2}$ to be continued through the replacement of eq. (2.8). On the other hand, it can be manipulated as follows

$$
\left(q \cdot \ell_{3}\right)\left(q \cdot \ell_{4}\right)=4\left(q \cdot \ell_{1}\right)\left(q \cdot \ell_{2}\right)-2 q^{2}\left(\ell_{1} \cdot \ell_{2}\right),
$$

and now the shift of eq. (2.8) would produce a $\tilde{q}^{2}$ contribution, in disagreement with our previous finding. We therefore conclude that not enough information is present in 
the 4-dimensional part to reconstruct $\mathrm{R}_{2}$. This is the reason why one is forced to work analytically in $n$ dimensions to reconstruct the $\mathrm{R}_{2}$ contribution. ${ }^{1}$

Nevertheless, based on a simple reasoning, one argues that some gauge invariance properties of the 4-dimensional part of the amplitude should be transferred to $R_{2}$. In fact, for physical processes, the sum of $R_{1}+R_{2}$ is gauge invariant. On the other hand, $R_{1}$ can be fully reconstructed from the 4-dimensional, gauge invariant, $\mathrm{CC}$ part of the amplitude, meaning that, by changing gauge, the same expressions for $\mathrm{R}_{1}$ should be found, and, as a consequence, also the same result for $\mathrm{R}_{2}$. This should be off course only true for amplitudes with physical external particles, because different gauges may have, in general, a different content in terms unphysical external fields. Therefore one can conjecture that

The $\mathrm{R}_{2}$ part of a physical amplitude gives the same result when computed in any gauge. ${ }^{2}$

This conjecture, being rather strong, should be proved with an actual calculation. Unfortunately, such a calculation would require to extend the set of basic integrals in [25] to be able to deal with non-renormalizable gauges. That is beyond the scope of this work, and we leave it for a future publication.

In the present paper, we fix the gauge to be the the 't Hooft-Feynman one and we derive all of the effective Electroweak $R_{2}$ Feynman rules by applying the splittings of eq. (2.6) Feynman diagram by Feynman diagram. For the interested reader, explicit examples of this technique can be found in [25].

\section{Results}

In this section, we give the complete list of the effective Electroweak vertices contributing to $\mathrm{R}_{2}$ in the 't Hooft-Feynman gauge. ${ }^{3}$ A parameter $\lambda_{H V}$ is introduced in our formulae such that $\lambda_{H V}=1$ corresponds to the 't Hooft-Veltman scheme and $\lambda_{H V}=0$ to the FDH scheme of eq. (2.7). We used the Feynman rules given in [35] and our notations are as follows: $l_{1}=e, l_{2}=\mu, l_{3}=\tau, l_{4}=\nu_{e}, l_{5}=\nu_{\mu}, l_{6}=\nu_{\tau}$ and $q_{1}=d, q_{2}=u, q_{3}=s$, $q_{4}=c, q_{5}=b, q_{6}=t$. In addition, $e_{1}=e, e_{2}=\mu, e_{3}=\tau, \nu_{1}=\nu_{e}, \nu_{2}=\nu_{\mu}, \nu_{3}=\nu_{\tau}$ and $u_{1}=u, u_{2}=c, u_{3}=t, d_{1}=d, d_{2}=s, d_{3}=b$. When appearing as external particles, $l, \nu_{l}$, $u$ and $d$ stand for the three charged leptons, the three (massless) neutrinos, the three uptype quarks and the three down-type quarks, respectively. Effective vertices with external quarks are always understood to be diagonal in the color space. Finally, $N_{c o l}$ is the number of colors and $V_{u_{i} d_{j}}$ are CKM matrix elements. Occasionally, combinations such as

$$
\sum_{i, j=1}^{3}\left(V_{u_{i} d_{j}} V_{d_{j} u_{i}}^{\dagger}\right)=3 \quad \text { and } \quad \sum_{i=1}^{3} 1=3
$$

\footnotetext{
${ }^{1}$ In other approaches $[16,17]$, a numerical determination of the whole $\mathrm{R}$ contribution can be achieved, but at the price of explicitly computing numerically the amplitude in 4, 6 and 8 dimensions.

${ }^{2}$ This does not mean that the $R_{2}$ part of the Green functions satisfy the Ward identities separately from $\mathrm{R}_{1}$, as we have checked explicitly.

${ }^{3}$ They can be also found as a FORM $[33,34]$ output in http://www.ugr.es/local/pittau/CutTools.
} 
appear in our formulae. In such cases, we do not explicitly work out the sum in order to make our results also readable family by family.

A last comment is in order with respect to our treatment of $\gamma_{5}$ in vertices containing fermionic lines. When computing all contributing Feynman diagrams, we pick up a "special" vertex in the loop and anticommute all $\gamma_{5}$ 's to reach it before performing the $n$-dimensional algebra, and, when a trace is present, we start reading it from this vertex. This treatment produces, in general, a term proportional to the totally antisymmetric $\epsilon$ tensor, whose coefficient may be different depending on the choice of the "special" vertex. However, when summing over all quantum numbers of each fermionic family, we checked that all contributions proportional to $\epsilon$ cancel. In addition, we explicitly verified that our results satisfy the large set of Ward identities given in appendix A.

\subsection{Electroweak effective vertices with 2 external legs}

In this section, we give the complete list of the non vanishing 2-point $R_{2}$ effective vertices.

\subsubsection{Scalar-Scalar effective vertices}

The generic effective vertex is

$$
S_{1}-\cdots-\cdots S_{2}=\frac{i e^{2}}{16 \pi^{2} s_{w}^{2}} C
$$

with the actual values of $S_{1}, S_{2}$ and $C$

$$
\begin{aligned}
H \chi: C= & 0 \\
H H: C= & \frac{m_{\phi}^{2}}{4}+\frac{m_{\chi}^{2}}{8 c_{w}^{2}}+\frac{1-12 \lambda_{H V}}{4}\left(1+\frac{1}{2 c_{w}^{4}}\right) m_{W}^{2}-\left(1+\frac{1}{2 c_{w}^{2}}\right) \frac{p^{2}}{12}+K \\
\chi \chi: C= & \frac{m_{\phi}^{2}}{4}+\frac{m_{H}^{2}}{8 c_{w}^{2}}+\frac{1-4 \lambda_{H V}}{4}\left(1+\frac{1}{2 c_{w}^{4}}\right) m_{W}^{2}-\left(1+\frac{1}{2 c_{w}^{2}}\right) \frac{p^{2}}{12}+K \\
\phi^{-} \phi^{+}: C= & \frac{m_{H}^{2}+m_{\chi}^{2}}{8}+\frac{\left(3-4 \lambda_{H V}\right) c_{w}^{4}-2 c_{w}^{2}+\left(\frac{1}{2}-2 \lambda_{H V}\right)}{c_{w}^{4}} \frac{m_{W}^{2}}{4}+\frac{m_{\phi}^{2}}{8 c_{w}^{2}} \\
& -\left(1+\frac{1}{2 c_{w}^{2}}\right) \frac{p^{2}}{12}+\frac{1}{2 m_{W}^{2}}\left[\sum_{i=1}^{3}\left(m_{e_{i}}^{2}\left(m_{e_{i}}^{2}-\frac{p^{2}}{3}\right)\right)\right. \\
& \left.+N_{\mathrm{col}} \sum_{i, j=1}^{3}\left(V_{u_{i} d_{j}} V_{d_{j} u_{i}}^{\dagger}\left(m_{u_{i}}^{2}+m_{d_{j}}^{2}\right)\left(m_{u_{i}}^{2}+m_{d_{j}}^{2}-\frac{p^{2}}{3}\right)\right)\right]
\end{aligned}
$$

where

$$
K=\frac{1}{m_{W}^{2}}\left[\sum_{i=1}^{6}\left(m_{l_{i}}^{2}\left(m_{l_{i}}^{2}-\frac{p^{2}}{6}\right)\right)+N_{\text {col }} \sum_{i=1}^{6}\left(m_{q_{i}}^{2}\left(m_{q_{i}}^{2}-\frac{p^{2}}{6}\right)\right)\right]
$$




\subsubsection{Vector-Vector effective vertices}

The generic effective vertex is

$$
V_{1 \alpha} \stackrel{p}{\sim} \sim V_{2 \beta}=\frac{i e^{2}}{\pi^{2}}\left(C_{1} p_{\alpha} p_{\beta}+C_{2} g_{\alpha \beta}\right)
$$

with the actual values of $V_{1}, V_{2}, C_{1}$ and $C_{2}$

$$
\begin{aligned}
A A: C_{1}= & -\frac{1}{24} \lambda_{H V} \\
C_{2}= & \frac{1}{8}\left[p^{2}\left(\frac{1}{6}+\frac{\lambda_{H V}}{3}\right)-m_{W}^{2}\right]-\frac{1}{4}\left[\sum_{i=1}^{6}\left(Q_{l_{i}}^{2}\left(m_{l_{i}}^{2}-\frac{1}{6} p^{2}\right)\right)\right. \\
& \left.+N_{\mathrm{col}} \sum_{i=1}^{6}\left(Q_{q_{i}}^{2}\left(m_{q_{i}}^{2}-\frac{1}{6} p^{2}\right)\right)\right] \\
A Z: C_{1}= & \frac{1}{24} \frac{c_{w}}{s_{w}} \lambda_{H V} \\
C_{2}= & -\frac{1}{8} \frac{c_{w}}{s_{w}}\left[p^{2}\left(\frac{1}{6}+\frac{\lambda_{H V}}{3}\right)-m_{W}^{2}\right]+\frac{1}{4 c_{w}}\left[\sum _ { i = 1 } ^ { 6 } \left(\left(\frac{Q_{l_{i}} I_{3 l_{i}}}{2 s_{w}}-Q_{l_{i}}^{2} s_{w}\right)\right.\right. \\
& \left.\left.\times\left(m_{l_{i}}^{2}-\frac{1}{6} p^{2}\right)\right)+N_{\mathrm{col}} \sum_{i=1}^{6}\left(\left(\frac{Q_{q_{i}} I_{3 q_{i}}}{2 s_{w}}-Q_{q_{i}}^{2} s_{w}\right)\left(m_{q_{i}}^{2}-\frac{1}{6} p^{2}\right)\right)\right] \\
Z Z: C_{1}= & \left.-\frac{1}{24} \frac{c_{w}^{2}}{s_{w}^{2}} \lambda_{H V}\right) \\
C_{2}= & \frac{1}{8} \frac{c_{w}^{2}}{s_{w}^{2}}\left[p^{2}\left(\frac{1}{6}+\frac{\lambda_{H V}}{3}\right)-m_{W}^{2}\right]+\frac{1}{4 c_{w}^{2}}\left[\sum _ { i = 1 } ^ { 6 } \left(\left(Q_{l_{i}} I_{3 l_{i}}-\frac{I_{3 l_{i}}^{2}}{2 s_{w}^{2}}-Q_{l_{i}}^{2} s_{w}^{2}\right)\right.\right. \\
& \left.\left.\times\left(m_{l_{i}}^{2}-\frac{1}{6} p^{2}\right)\right)+N_{\mathrm{col}} \sum_{i=1}^{6}\left(\left(Q_{q_{i}} I_{3 q_{i}}-\frac{I_{3 q_{i}}^{2}}{2 s_{w}^{2}}-Q_{q_{i}}^{2} s_{w}^{2}\right)\left(m_{q_{i}}^{2}-\frac{1}{6} p^{2}\right)\right)\right] \\
& \left.+\frac{1}{24 s_{w}^{2}} \lambda_{H V} \sum_{i, j=1}^{3}\left(V_{u_{i} d_{j}} V_{d_{j} u_{i}}^{\dagger}\left(m_{u_{i}}^{2}+m_{d_{j}}^{2}-\frac{p^{2}}{3}\right)\right)\right] \\
C^{-} W^{+}: C_{1}= & \frac{1}{8 s_{w}^{2}}\left[p^{2}\left(\frac{1}{6}+\frac{\lambda_{H V}}{3}\right)-m_{W}^{2}\right]-\frac{1}{32 s_{w}^{2}}\left[\sum_{i=1}^{3}\left(m_{e_{i}}^{2}-\frac{p^{2}}{3}\right)\right. \\
C_{2} & \\
C_{2} &
\end{aligned}
$$

\subsubsection{Fermion-Fermion effective vertices}

The generic effective vertex is

$$
F_{1} \stackrel{p}{\longrightarrow} \bullet \bar{F}_{2}=\frac{i e^{2}}{\pi^{2}}\left[\left(C_{-} \Omega^{-}+C_{+} \Omega^{+}\right) \not p+C_{0}\right] \lambda_{H V}
$$


with the actual values of $F_{1}, \bar{F}_{2}, C_{-}, C_{+}$and $C_{0}$

$$
\begin{aligned}
u \bar{u}: C_{-} & =\frac{1}{16} \frac{Q_{u}^{2}}{c_{w}^{2}} \\
C_{+} & =\frac{1}{16}\left(\frac{I_{3 u}^{2}}{s_{w}^{2} c_{w}^{2}}-\frac{2 Q_{u} I_{3 u}}{c_{w}^{2}}+\frac{Q_{u}^{2}}{c_{w}^{2}}+\frac{1}{2 s_{w}^{2}} \sum_{j=1}^{3}\left(V_{u d_{j}} V_{d_{j} u}^{\dagger}\right)\right) \\
C_{0} & =\frac{m_{u} Q_{u}}{8 c_{w}^{2}}\left(Q_{u}-I_{3 u}\right) \\
d \bar{d} \quad: \quad C_{-} & =\frac{1}{16} \frac{Q_{d}^{2}}{c_{w}^{2}} \\
C_{+} & =\frac{1}{16}\left(\frac{I_{3 d}^{2}}{s_{w}^{2} c_{w}^{2}}-\frac{2 Q_{d} I_{3 d}}{c_{w}^{2}}+\frac{Q_{d}^{2}}{c_{w}^{2}}+\frac{1}{2 s_{w}^{2}} \sum_{i=1}^{3}\left(V_{u_{i} d} V_{d u_{i}}^{\dagger}\right)\right) \\
C_{0} & =\frac{m_{d} Q_{d}}{8 c_{w}^{2}}\left(Q_{d}-I_{3 d}\right) \\
l \bar{l} \quad: \quad C_{-} & =\frac{1}{16} \frac{Q_{l}^{2}}{c_{w}^{2}} \\
C_{+} & =\frac{1}{16}\left(\frac{I_{3 l}^{2}}{s_{w}^{2} c_{w}^{2}}-\frac{2 Q_{l} I_{3 l}}{c_{w}^{2}}+\frac{Q_{l}^{2}}{c_{w}^{2}}+\frac{1}{2 s_{w}^{2}}\right) \\
C_{0} & =\frac{m_{l} Q_{l}}{8 c_{w}^{2}}\left(Q_{l}-I_{3 l}\right) \\
\nu_{l} \bar{\nu}_{l} \quad: \quad C_{-} & =0 \\
C_{+} & =\frac{1}{32 s_{w}^{2}}\left(\frac{1}{2 c_{w}^{2}}+1\right) \\
C_{0} & =0
\end{aligned}
$$

\subsection{Electroweak effective vertices with 3 external legs}

We list here the 3 -point $R_{2}$ effective vertices.

\subsubsection{Scalar-Fermion-Fermion effective vertices}

The generic effective vertex is

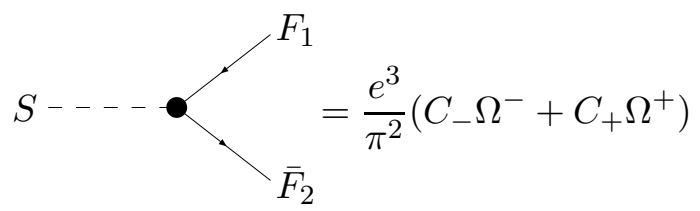

with the actual values of $S, F_{1}, \bar{F}_{2}, C_{-}$and $C_{+}$

$$
\begin{aligned}
H u \bar{u}: C_{-} & =\frac{i m_{u}}{8 m_{W} s_{w}}\left[\frac{\left(1+\lambda_{H V}\right) Q_{u}^{2}}{2 c_{w}^{2}}+\frac{1}{16 s_{w}^{2}} \sum_{j=1}^{3}\left(V_{u d_{j}} V_{d_{j} u}^{\dagger}\right)+\frac{I_{3 u}}{c_{w}^{2}}\left(\frac{I_{3 u}}{8 s_{w}^{2}}\right.\right. \\
& \left.\left.-\frac{\left(1+\lambda_{H V}\right) Q_{u}}{2}\right)+\frac{1}{16 m_{W}^{2} s_{w}^{2}} \sum_{j=1}^{3}\left(m_{d_{j}}^{2} V_{u d_{j}} V_{d_{j} u}^{\dagger}\right)\right]
\end{aligned}
$$




$$
\begin{aligned}
& C_{+}=C_{-} \\
& H d \bar{d}: C_{-}=\frac{i m_{d}}{8 m_{W} s_{w}}\left[\frac{\left(1+\lambda_{H V}\right) Q_{d}^{2}}{2 c_{w}^{2}}+\frac{1}{16 s_{w}^{2}} \sum_{i=1}^{3}\left(V_{u_{i} d} V_{d u_{i}}^{\dagger}\right)+\frac{I_{3 d}}{c_{w}^{2}}\left(\frac{I_{3 d}}{8 s_{w}^{2}}\right.\right. \\
& \left.\left.-\frac{\left(1+\lambda_{H V}\right) Q_{d}}{2}\right)+\frac{1}{16 m_{W}^{2} s_{w}^{2}} \sum_{i=1}^{3}\left(m_{u_{i}}^{2} V_{u_{i} d} V_{d u_{i}}^{\dagger}\right)\right] \\
& C_{+}=C_{-} \\
& H \bar{l} \bar{l}: C_{-}=\frac{i m_{l}}{8 m_{W} s_{w}}\left[\frac{\left(1+\lambda_{H V}\right) Q_{l}^{2}}{2 c_{w}^{2}}+\frac{1}{16 s_{w}^{2}}+\frac{I_{3 l}}{c_{w}^{2}}\left(\frac{I_{3 l}}{8 s_{w}^{2}}-\frac{\left(1+\lambda_{H V}\right) Q_{l}}{2}\right)\right] \\
& C_{+}=C_{-} \\
& H \nu_{l} \bar{\nu}_{l} \quad: \quad C_{-}=0 \\
& C_{+}=0 \\
& \chi u \bar{u}: C_{-}=-\frac{m_{u}}{4 m_{W} s_{w}}\left[\frac{\left(1+\lambda_{H V}\right) Q_{u}^{2} I_{3 u}}{2 c_{w}^{2}}+\frac{1}{32 s_{w}^{2}} \sum_{j=1}^{3}\left(V_{u d_{j}} V_{d_{j} u}^{\dagger}\right)+\frac{I_{3 u}}{c_{w}^{2}}\left(\frac{1}{32 s_{w}^{2}}\right.\right. \\
& \left.\left.-\frac{\left(1+\lambda_{H V}\right) Q_{u} I_{3 u}}{2}\right)-\frac{1}{16 m_{W}^{2} s_{w}^{2}} \sum_{j=1}^{3}\left(m_{d_{j}}^{2} I_{3 d_{j}} V_{u d_{j}} V_{d_{j} u}^{\dagger}\right)\right] \\
& C_{+}=-C_{-} \\
& \chi d \bar{d}: C_{-}=-\frac{m_{d}}{4 m_{W} s_{w}}\left[\frac{\left(1+\lambda_{H V}\right) Q_{d}^{2} I_{3 d}}{2 c_{w}^{2}}-\frac{1}{32 s_{w}^{2}} \sum_{i=1}^{3}\left(V_{u_{i} d} V_{d u_{i}}^{\dagger}\right)+\frac{I_{3 d}}{c_{w}^{2}}\left(\frac{1}{32 s_{w}^{2}}\right.\right. \\
& \left.\left.-\frac{\left(1+\lambda_{H V}\right) Q_{d} I_{3 d}}{2}\right)-\frac{1}{16 m_{W}^{2} s_{w}^{2}} \sum_{i=1}^{3}\left(m_{u_{i}}^{2} I_{3 u_{i}} V_{u_{i} d} V_{d u_{i}}^{\dagger}\right)\right] \\
& C_{+}=-C_{-} \\
& \chi l \bar{l}: C_{-}=-\frac{m_{l}}{4 m_{W} s_{w}}\left[\frac{\left(1+\lambda_{H V}\right) Q_{l}^{2} I_{3 l}}{2 c_{w}^{2}}-\frac{1}{32 s_{w}^{2}}+\frac{I_{3 l}}{c_{w}^{2}}\left(\frac{1}{32 s_{w}^{2}}-\frac{\left(1+\lambda_{H V}\right) Q_{l} I_{3 l}}{2}\right)\right. \\
& \left.-\frac{m_{l}^{2} I_{3 l}}{8 m_{W}^{2} s_{w}^{2}}\left(-\frac{1}{4}+I_{3 l}^{2}\right)\right] \\
& C_{+}=-C_{-} \\
& \chi \nu_{l} \bar{\nu}_{l}: C_{-}=0 \\
& C_{+}=0 \\
& \phi^{-} u \bar{d}: C_{-}=-\frac{i m_{d} V_{d u}^{\dagger}}{4 \sqrt{2} m_{W} s_{w}}\left[\frac{1}{c_{w}^{2}}\left(\frac{-1}{16}-\frac{\left(1+\lambda_{H V}\right) Q_{u} Q_{d}}{2}\right)-\frac{3}{32 s_{w}^{2}}\right. \\
& \left.-\frac{m_{u}^{2}}{16 s_{w}^{2} m_{W}^{2}}+\frac{I_{3 u}}{c_{w}^{2}}\left(\frac{\left(1+\lambda_{H V}\right) Q_{d}}{2}+\frac{1}{16}\right)\right] \\
& C_{+}=\frac{i m_{u} V_{d u}^{\dagger}}{4 \sqrt{2} m_{W} s_{w}}\left[\frac{1}{c_{w}^{2}}\left(\frac{-1}{16}-\frac{\left(1+\lambda_{H V}\right) Q_{u} Q_{d}}{2}\right)-\frac{3}{32 s_{w}^{2}}\right. \\
& \left.-\frac{m_{d}^{2}}{16 s_{w}^{2} m_{W}^{2}}+\frac{I_{3 d}}{c_{w}^{2}}\left(\frac{\left(1+\lambda_{H V}\right) Q_{u}}{2}-\frac{1}{16}\right)\right]
\end{aligned}
$$




$$
\begin{aligned}
\phi^{+} d \bar{u}: \quad C_{-}= & -\frac{i m_{u} V_{u d}}{4 \sqrt{2} s_{w} m_{W}}\left[\frac{1}{c_{w}^{2}}\left(\frac{1}{16}+\frac{\left(1+\lambda_{H V}\right) Q_{u} Q_{d}}{2}\right)+\frac{3}{32 s_{w}^{2}}\right. \\
& \left.+\frac{m_{d}^{2}}{16 s_{w}^{2} m_{W}^{2}}-\frac{I_{3 d}}{c_{w}^{2}}\left(\frac{\left(1+\lambda_{H V}\right) Q_{u}}{2}-\frac{1}{16}\right)\right] \\
C_{+}= & \frac{i m_{d} V_{u d}}{4 \sqrt{2} m_{W} s_{w}}\left[\frac{1}{c_{w}^{2}}\left(\frac{1}{16}+\frac{\left(1+\lambda_{H V}\right) Q_{u} Q_{d}}{2}\right)+\frac{3}{32 s_{w}^{2}}\right. \\
& \left.+\frac{m_{u}^{2}}{16 s_{w}^{2} m_{W}^{2}}-\frac{I_{3 u}}{c_{w}^{2}}\left(\frac{\left(1+\lambda_{H V}\right) Q_{d}}{2}+\frac{1}{16}\right)\right] \\
\phi^{-} \nu_{l} \bar{l}: C_{-}= & -\frac{i m_{l}}{4 \sqrt{2} m_{W} s_{w}}\left[\frac{Q_{l}}{16 c_{w}^{2}}-\frac{3}{32 s_{w}^{2}}+\frac{I_{3 \nu_{l}}}{c_{w}^{2}}\left(\frac{\left(1+\lambda_{H V}\right) Q_{l}}{2}+\frac{1}{16}\right)\right] \\
\phi^{+} l \bar{\nu}_{l}: C_{+}= & 0 \\
C_{-}= & 0 \\
C_{+}= & \frac{i m_{l}}{4 \sqrt{2} m_{W} s_{w}}\left[-\frac{Q_{l}}{16 c_{w}^{2}}+\frac{3}{32 s_{w}^{2}}-\frac{I_{3 \nu_{l}}}{c_{w}^{2}}\left(\frac{\left(1+\lambda_{H V}\right) Q_{l}}{2}+\frac{1}{16}\right)\right]
\end{aligned}
$$

\subsubsection{Vector-Fermion-Fermion effective vertices}

The generic effective vertex is

$$
V_{\mu} \sim_{\bar{F}_{2}}^{F_{1}}=\frac{i e^{3}}{\pi^{2}}\left(C_{-} \Omega^{-}+C_{+} \Omega^{+}\right) \gamma_{\mu}
$$

with the actual values of $V, F_{1}, \bar{F}_{2}, C_{-}$and $C_{+}$

$$
\begin{aligned}
A u \bar{u}: C_{-}= & \frac{1}{4}\left[\frac{\left(1+\lambda_{H V}\right) Q_{u}^{3}}{4 c_{w}^{2}}+\frac{m_{u}^{2}}{8 s_{w}^{2} m_{W}^{2}}\left(\frac{1}{2} \sum_{j=1}^{3}\left(V_{u d_{j}} V_{d_{j} u}^{\dagger} Q_{d_{j}}\right)\right.\right. \\
& \left.\left.+\frac{Q_{u}}{4}+Q_{u} I_{3 u}^{2}\right)\right] \\
C_{+}= & \frac{1}{4}\left[\frac{\left(1+\lambda_{H V}\right) Q_{u}^{3}}{4 c_{w}^{2}}-\frac{\left(1+\lambda_{H V}\right) Q_{u}^{2} I_{3 u}}{2 c_{w}^{2}}+\frac{\left(1+\lambda_{H V}\right) Q_{u} I_{3 u}^{2}}{4 s_{w}^{2} c_{w}^{2}}\right. \\
& +\frac{1}{4 s_{w}^{2}}\left(\frac{1}{4 m_{W}^{2}} \sum_{j=1}^{3}\left(V_{u d_{j}} V_{d_{j} u}^{\dagger} m_{d_{j}}^{2} Q_{d_{j}}\right)\right. \\
& \left.\left.+\frac{m_{u}^{2} Q_{u}\left(1+4 I_{3 u}^{2}\right)}{8 m_{W}^{2}}+\sum_{j=1}^{3}\left(V_{u d_{j}} V_{d_{j} u}^{\dagger}\left(1+Q_{d_{j}}\right) \frac{\left(1+\lambda_{H V}\right)}{2}\right)\right)\right] \\
A d \bar{d}: C_{-}= & \frac{1}{4}\left[\frac{\left(1+\lambda_{H V}\right) Q_{d}^{3}}{4 c_{w}^{2}}+\frac{m_{d}^{2}}{8 s_{w}^{2} m_{W}^{2}}\left(\frac{1}{2} \sum_{i=1}^{3}\left(V_{u_{i} d} V_{d u_{i}}^{\dagger} Q_{u_{i}}\right)\right.\right. \\
& \left.\left.+\frac{Q_{d}}{4}+Q_{d} I_{3 d}^{2}\right)\right]
\end{aligned}
$$




$$
\begin{aligned}
C_{+}= & \frac{1}{4}\left[\frac{\left(1+\lambda_{H V}\right) Q_{d}^{3}}{4 c_{w}^{2}}-\frac{\left(1+\lambda_{H V}\right) Q_{d}^{2} I_{3 d}}{2 c_{w}^{2}}+\frac{\left(1+\lambda_{H V}\right) Q_{d} I_{3 d}^{2}}{4 s_{w}^{2} c_{w}^{2}}\right. \\
& +\frac{1}{4 s_{w}^{2}}\left(\frac{1}{4 m_{W}^{2}} \sum_{i=1}^{3}\left(V_{u_{i} d} V_{d u_{i}}^{\dagger} m_{u_{i}}^{2} Q_{u_{i}}\right)\right. \\
& \left.\left.+\frac{m_{d}^{2} Q_{d}\left(1+4 I_{3 d}^{2}\right)}{8 m_{W}^{2}}+\sum_{i=1}^{3}\left(V_{u_{i} d} V_{d u_{i}}^{\dagger}\left(Q_{u_{i}}-1\right) \frac{\left(1+\lambda_{H V}\right)}{2}\right)\right)\right] \\
A l \bar{l}: C_{-}= & \frac{1}{4}\left[\frac{\left(1+\lambda_{H V}\right) Q_{l}^{3}}{4 c_{w}^{2}}+\frac{m_{l}^{2}}{8 s_{w}^{2} m_{W}^{2}}\left(\frac{Q_{l}}{4}+Q_{l} I_{3 l}^{2}\right)\right] \\
C_{+}= & \frac{1}{4}\left[\frac{\left(1+\lambda_{H V}\right) Q_{l}^{3}}{4 c_{w}^{2}}-\frac{\left(1+\lambda_{H V}\right) Q_{l}^{2} I_{3 l}}{2 c_{w}^{2}}+\frac{\left(1+\lambda_{H V}\right) Q_{l} I_{3 l}^{2}}{4 s_{w}^{2} c_{w}^{2}}\right. \\
& \left.+\frac{1}{4 s_{w}^{2}}\left(\frac{m_{l}^{2} Q_{l}\left(1+4 I_{3 l}^{2}\right)}{8 m_{W}^{2}}-1\right) \frac{\left(1+\lambda_{H V}\right)}{2}\right]
\end{aligned}
$$

$A \nu_{l} \bar{\nu}_{l}: C_{-}=0$

$$
\begin{aligned}
C_{+}= & \frac{1}{32 s_{w}^{2}}\left[\frac{m_{l}^{2} Q_{l}}{2 m_{W}^{2}}+\left(Q_{l}+1\right)\left(1+\lambda_{H V}\right)\right] \\
Z u \bar{u}: C_{-}= & \frac{1}{8 c_{w}}\left\{\frac{\left(1+\lambda_{H V}\right) Q_{u}^{3} s_{w}}{2 c_{w}^{2}}+\frac{m_{u}^{2}}{8 s_{w} m_{W}^{2}}\left[\sum_{j=1}^{3}\left(V_{u d_{j}} V_{d_{j} u}^{\dagger}\left(Q_{d_{j}}-\frac{I_{3 d_{j}}}{s_{w}^{2}}\right)\right)\right.\right. \\
& \left.\left.+\left(Q_{u}-\frac{I_{3 u}}{s_{w}^{2}}\right)\right]\right\} \\
C_{+}= & \frac{1}{8 c_{w}}\left\{\frac{\left(1+\lambda_{H V}\right) Q_{u}^{3} s_{w}}{2 c_{w}^{2}}-\frac{\left(1+\lambda_{H V}\right) Q_{u}^{2} I_{3 u}\left(1+2 s_{w}^{2}\right)}{2 s_{w} c_{w}^{2}}\right. \\
& +3 \frac{\left(1+\lambda_{H V}\right) Q_{u} I_{3 u}^{2}}{2 s_{w} c_{w}^{2}}-\frac{\left(1+\lambda_{H V}\right) I_{3 u}^{3}}{2 s_{w}^{3} c_{w}^{2}} \\
& +\frac{1}{2 s_{w}}\left[\frac{1}{4 m_{W}^{2}}\left(\sum_{j=1}^{3}\left(V_{u d_{j}} V_{d_{j} u}^{\dagger} m_{d_{j}}^{2} Q_{d_{j}}\right)+\frac{m_{u}^{2} Q_{u}\left(1+4 I_{3 u}^{2}\right)}{2}\right)\right. \\
& \left.\left.+\sum_{j=1}^{3}\left(V_{u d_{j}} V_{d_{j} u}^{\dagger} \frac{\left(1+\lambda_{H V}\right)}{2}\left(Q_{d_{j}}-\frac{c_{w}^{2}+I_{3 d_{j}}}{s_{w}^{2}}\right)\right)\right]\right\} \\
Z d \bar{d}: C_{-}= & \frac{1}{8 c_{w}}\left\{\frac{\left(1+\lambda_{H V}\right) Q_{d}^{3} s_{w}}{2 c_{w}^{2}}+\frac{m_{d}^{2}}{8 s_{w} m_{W}^{2}}\left[\sum_{i=1}^{3}\left(V_{u_{i} d} V_{d u_{i}}^{\dagger}\left(Q_{u_{i}}-\frac{I_{3 u_{i}}}{s_{w}^{2}}\right)\right)\right.\right. \\
& \left.\left.+\left(Q_{d}-\frac{I_{3 d}}{s_{w}^{2}}\right)\right]\right\} \\
& +\frac{1}{s_{w}}\left[\frac{1}{4 m_{W}^{2}}\left(\sum_{i=1}^{3}\left(V_{u_{i} d} V_{d u_{i}}^{\dagger} m_{u_{i}}^{2} Q_{u_{i}}\right)+\frac{m_{d}^{2} Q_{d}\left(1+4 I_{3 d}^{2}\right)}{2}\right)\right. \\
C_{+}= & \frac{1}{16 c_{w}}\left\{\left(1+\lambda_{H V}\right)\left(\frac{Q_{d}^{3} s_{w}}{c_{w}^{2}}-\frac{Q_{d}^{2} I_{3 d}\left(1+2 s_{w}^{2}\right)}{s_{w} c_{w}^{2}}+3 \frac{Q_{d} I_{3 d}^{2}}{s_{w} c_{w}^{2}}-\frac{I_{3 d}^{3}}{s_{w}^{3} c_{w}^{2}}\right)\right. \\
& \\
C_{w} & \\
&
\end{aligned}
$$




$$
\begin{aligned}
&\left.+\sum_{i=1}^{3}\left(\frac{1+\lambda_{H V}}{2}\right)\left(V_{u_{i} d} V_{d u_{i}}^{\dagger}\left(Q_{u_{i}}+\frac{\left.\left.\left.\left.c_{w}^{2}-I_{3 u_{i}}\right)\right)\right]\right\}}{s_{w}^{2}}\right)\right]\right\} \\
& Z l \bar{l}: C_{-}= \frac{1}{8 c_{w}}\left\{\frac{\left(1+\lambda_{H V}\right) Q_{l}^{3} s_{w}}{2 c_{w}^{2}}+\frac{m_{l}^{2}}{4 s_{w} m_{W}^{2}}\left[-\frac{1}{4 s_{w}^{2}}+\frac{1}{2}\left(Q_{l}-\frac{I_{3 l}}{s_{w}^{2}}\right)\right]\right\} \\
& C_{+}= \frac{1}{16 c_{w}}\left\{\left(\frac{Q_{l}^{3} s_{w}}{c_{w}^{2}}-\frac{Q_{l}^{2} I_{3 l}\left(1+2 s_{w}^{2}\right)}{s_{w} c_{w}^{2}}\right.\right. \\
&\left.+3 \frac{Q_{l} I_{3 l}^{2}}{s_{w} c_{w}^{2}}-\frac{I_{3 l}^{3}}{s_{w}^{3} c_{w}^{2}}\right)\left(1+\lambda_{H V}\right)+\frac{1}{2 s_{w}}\left[\frac{m_{l}^{2} Q_{l}}{2 m_{W}^{2}}\right. \\
&\left.\left.+\frac{1}{s_{w}^{2}}\left(1+\lambda_{H V}\right)\left(c_{w}^{2}-I_{3 \nu_{l}}\right)\right]\right\} \\
& Z \nu_{l} \bar{\nu}_{l}: C_{-}= 0 \\
& C_{+}= \frac{1}{16 c_{w}}\left\{-\frac{\left(1+\lambda_{H V}\right) I_{3 \nu_{l}}^{3}}{s_{w}^{3} c_{w}^{2}}+\frac{1}{2 s_{w}}\left[\frac{m_{l}^{2} Q_{l}}{2 m_{W}^{2}}\right.\right. \\
&\left.\left.+\left(1+\lambda_{H V}\right)\left(Q_{l}-\frac{c_{w}^{2}+I_{3 l}}{s_{w}^{2}}\right)\right]\right\} \\
& W^{-} u \bar{d}: C_{-}= 0 \\
& C_{+}= \frac{V_{d u}^{\dagger}}{16 \sqrt{2} s_{w}}\left[\frac{Q_{d} I_{3 u}+Q_{u} I_{3 d}-Q_{u} Q_{d}}{c_{w}^{2}}-\frac{1}{s_{w}^{2}}+\frac{1}{4 s_{w}^{2} c_{w}^{2}}\right]\left(1+\lambda_{H V}\right) \\
& W^{+} d \bar{u}: C_{-}= 0 \\
& C_{+}= \frac{V_{u d}}{16 \sqrt{2} s_{w}}\left[\frac{Q_{d} I_{3 u}+Q_{u} I_{3 d}-Q_{u} Q_{d}}{c_{w}^{2}}-\frac{1}{s_{w}^{2}}+\frac{1}{4 s_{w}^{2} c_{w}^{2}}\right]\left(1+\lambda_{H V}\right) \\
& W^{-} \nu_{l} \bar{l} \\
&\left.W^{+} l \bar{\nu}_{l}\right\}: C_{-}= 0 \\
& C_{+}= \frac{1}{16 \sqrt{2} s_{w}}\left[\frac{Q_{l} I_{3 \nu_{l}}}{c_{w}^{2}}-\frac{1}{s_{w}^{2}}+\frac{1}{4 s_{w}^{2} c_{w}^{2}}\right]\left(1+\lambda_{H V}\right)
\end{aligned}
$$

\subsubsection{Scalar-Scalar-Scalar effective vertices}

The generic effective vertex is

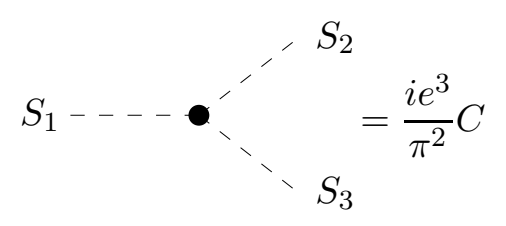

with the actual values of $S_{1}, S_{2}, S_{3}$, and $C$

$$
\left.\begin{array}{l}
H H \chi \\
\chi \chi \chi \\
\chi \phi^{+} \phi^{-}
\end{array}\right\}: C=0
$$




$$
\begin{aligned}
H H H: C= & \frac{3}{32 s_{w}^{3}}\left[\frac{1-4 \lambda_{H V}}{2} m_{W}+\frac{1}{m_{W}^{3}}\left(\sum_{i=1}^{6} m_{l_{i}}^{4}+N_{\text {col }} \sum_{i=1}^{6} m_{q_{i}}^{4}\right)\right. \\
& \left.+\frac{1}{4}\left(1+\frac{1}{2 c_{w}^{2}}\right) \frac{m_{H}^{2}}{m_{W}}+\frac{\left(1-4 \lambda_{H V}\right) m_{W}}{4 c_{w}^{4}}\right] \\
H \chi \chi: C= & \frac{1}{8 s_{w}^{3}}\left[\frac{1-4 \lambda_{H V}}{8} m_{W}+\frac{1}{4 m_{W}^{3}}\left(\sum_{i=1}^{6} m_{l_{i}}^{4}+N_{\mathrm{col}} \sum_{i=1}^{6} m_{q_{i}}^{4}\right)\right. \\
& \left.+\frac{1}{16}\left(1+\frac{1}{2 c_{w}^{2}}\right) \frac{m_{H}^{2}}{m_{W}}+\frac{\left(1-4 \lambda_{H V}\right) m_{W}}{16 c_{w}^{4}}\right] \\
H \phi^{+} \phi^{-}: \quad C= & \frac{1}{32 s_{w}^{3}}\left[\frac{1}{m_{W}^{3}}\left(\sum_{i=1}^{3} m_{e_{i}}^{4}+N_{\mathrm{col}} \sum_{i, j=1}^{3}\left(V_{u_{i} d_{j}} V_{d_{j} u_{i}}^{\dagger}\left(m_{u_{i}}^{4}+m_{d_{j}}^{4}\right)\right)\right)\right. \\
& \left.+\frac{\left(1+2 c_{w}^{2}\right)}{8 c_{w}^{2}} \frac{m_{H}^{2}}{m_{W}}+\frac{3\left(1-4 \lambda_{H V}\right)}{4} m_{W}+\frac{1-4 \lambda_{H V}}{4} \frac{s_{w}^{2}\left(1+c_{w}^{2}\right)}{c_{w}^{4}} m_{W}\right]
\end{aligned}
$$

\subsubsection{Vector-Scalar-Scalar effective vertices}

The generic effective vertex is

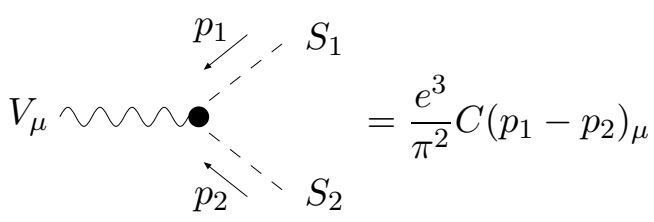

with the actual values of $V, S_{1}, S_{2}$, and $C$

$$
\begin{aligned}
& \left.\begin{array}{l}
A H H \\
Z H H \\
A \chi \chi \\
Z \chi \chi
\end{array}\right\}: C=0 \\
& A \chi H \quad: \quad C=\frac{5}{192 s_{w}^{2}} \\
& Z \chi H \quad: \quad C=-\frac{1}{96 s_{w} c_{w}}\left[\frac{1+2 c_{w}^{2}+20 c_{w}^{4}}{8 s_{w}^{2} c_{w}^{2}}+\frac{1}{s_{w}^{2} m_{W}^{2}}\left(\sum_{i=1}^{6}\left(m_{l_{i}}^{2}+N_{\mathrm{col}} m_{q_{i}}^{2}\right)\right)\right] \\
& A \phi^{+} \phi^{-}: C=\frac{i}{48 s_{w}^{2}}\left[\frac{1+12 c_{w}^{2}}{8 c_{w}^{2}}+\frac{1}{m_{W}^{2}}\left(-\sum_{i=1}^{3}\left(m_{e_{i}}^{2} Q_{e_{i}}\right)\right.\right. \\
& \left.\left.+N_{\mathrm{col}} \sum_{i, j=1}^{3}\left(V_{u_{i} d_{j}} V_{d_{j} u_{i}}^{\dagger}\left(m_{u_{i}}^{2}+m_{d_{j}}^{2}\right)\right)\right)\right]
\end{aligned}
$$




$$
\left.\left.\begin{array}{rl}
Z \phi^{+} \phi^{-}: \quad C= & \frac{i}{48 s_{w} c_{w}}\left[\frac{1-24 c_{w}^{4}}{16 c_{w}^{2} s_{w}^{2}}+\frac{1}{m_{W}^{2}}\left(-\sum_{i=1}^{3}\left(m_{e_{i}}^{2}\left(Q_{e_{i}}+I_{3 \nu_{i}}\right)\right)\right.\right. \\
& \left.\left.+N_{\text {col }} \sum_{i, j=1}^{3}\left(V_{u_{i} d_{j}} V_{d_{j} u_{i}}^{\dagger}\left(m_{u_{i}}^{2}+m_{d_{j}}^{2}\right)+m_{u_{i}}^{2} I_{3 d_{j}}-m_{d_{i}}^{2} I_{3 u_{i}}\right)\right)\right] \\
W^{+} \phi^{-} H \\
W^{-} H \phi^{+}
\end{array}\right\}: \begin{array}{rl}
C= & \frac{i}{96 s_{w}^{3}}\left[\frac{1+22 c_{w}^{2}}{8 c_{w}^{2}}+\frac{1}{m_{W}^{2}}\left(\sum_{i=1}^{3} m_{e_{i}}^{2}\right.\right. \\
& \left.\left.+N_{\mathrm{col}} \sum_{i, j=1}^{3}\left(V_{u_{i} d_{j}} V_{d_{j} u_{i}}^{\dagger}\left(m_{u_{i}}^{2}+m_{d_{j}}^{2}\right)\right)\right)\right] \\
W^{+} \phi^{-} \chi \\
W^{-} \phi^{+} \chi
\end{array}\right\}: \begin{aligned}
C= & \frac{1}{48 s_{w}^{3}}\left[-\frac{1+22 c_{w}^{2}}{16 c_{w}^{2}}+\frac{1}{m_{W}^{2}}\left(\sum_{i=1}^{3}\left(m_{e_{i}}^{2} I_{3 e_{i}}\right)\right.\right. \\
& \left.\left.-N_{\mathrm{col}} \sum_{i, j=1}^{3}\left(V_{u_{i} d_{j}} V_{d_{j} u_{i}}^{\dagger}\left(m_{u_{i}}^{2} I_{3 u_{i}}-m_{d_{j}}^{2} I_{3 d_{j}}\right)\right)\right)\right]
\end{aligned}
$$

\subsubsection{Scalar-Vector-Vector effective vertices}

The generic effective vertex is

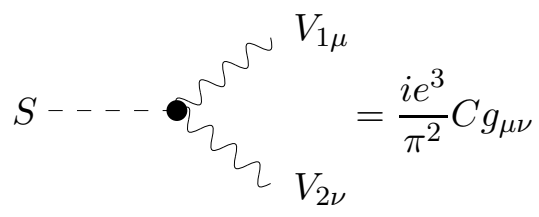

with the actual values of $S, V_{1}, V_{2}$ and $C$

$$
\begin{aligned}
& \left.\begin{array}{l}
\chi A A \\
\chi A Z \\
\chi Z Z \\
\chi W^{-} W^{+}
\end{array}\right\}: C=0 \\
& H A A: C=-\frac{1}{8 s_{w}}\left[\frac{1}{m_{W}}\left(\sum_{i=1}^{6}\left(m_{l_{i}}^{2} Q_{l_{i}}^{2}\right)+N_{\mathrm{col}} \sum_{i=1}^{6}\left(m_{q_{i}}^{2} Q_{q_{i}}^{2}\right)\right)+\frac{m_{W}}{2}\right] \\
& H A Z: C=\frac{1}{8 c_{w}}\left\{\frac { 1 } { m _ { W } } \left[\sum_{i=1}^{6}\left(m_{l_{i}}^{2} Q_{l_{i}}\left(\frac{I_{3 l_{i}}}{2 s_{w}^{2}}-Q_{l_{i}}\right)\right)\right.\right. \\
& \left.\left.+N_{\mathrm{col}} \sum_{i=1}^{6}\left(m_{q_{i}}^{2} Q_{q_{i}}\left(\frac{I_{3 q_{i}}}{2 s_{w}^{2}}-Q_{q_{i}}\right)\right)\right]+\frac{m_{W}\left(1+2 c_{w}^{2}\right)}{4 s_{w}^{2}}\right\} \\
& H Z Z: C=\frac{1}{8}\left\{\frac { 1 } { m _ { W } c _ { w } ^ { 2 } } \left[\sum_{i=1}^{6}\left(m_{l_{i}}^{2}\left(\frac{Q_{l_{i}} I_{3 l_{i}}}{s_{w}}-Q_{l_{i}}^{2} s_{w}-\frac{I_{3 l_{i}}^{2}}{s_{w}^{3}}\right)\right)\right.\right. \\
& \left.\left.+N_{\mathrm{col}} \sum_{i=1}^{6}\left(m_{q_{i}}^{2}\left(\frac{Q_{q_{i}} I_{3 q_{i}}}{s_{w}}-Q_{q_{i}}^{2} s_{w}-\frac{I_{3 q_{i}}^{2}}{s_{w}^{3}}\right)\right)\right]+\frac{m_{W}\left(s_{w}^{2}-2\right)}{2 s_{w}^{3}}\right\}
\end{aligned}
$$




$$
\begin{aligned}
& H W^{-} W^{+} \quad: \quad C=-\frac{1}{8 s_{w}^{3}}\left[\frac { 1 } { 4 m _ { W } } \left(\sum_{i=1}^{3} m_{e_{i}}^{2}\right.\right. \\
& \left.\left.+N_{\mathrm{col}} \sum_{i, j=1}^{3}\left(V_{u_{i} d_{j}} V_{d_{j} u_{i}}^{\dagger}\left(m_{u_{i}}^{2}+m_{d_{j}}^{2}\right)\right)\right)+m_{W}\right] \\
& \left.\begin{array}{l}
\phi^{-} A W^{+} \\
\phi^{+} W^{-} A
\end{array}\right\}: \quad C=\frac{1}{32 s_{w}^{2}} K \\
& \left.\begin{array}{l}
\phi^{-} Z W^{+} \\
\phi^{+} W^{-} Z
\end{array}\right\}: C=\frac{1}{32 s_{w} c_{w}} K
\end{aligned}
$$

where

$$
K=m_{W}+\frac{N_{\mathrm{col}}}{m_{W}} \sum_{i, j=1}^{3}\left(V_{u_{i} d_{j}} V_{d_{j} u_{i}}^{\dagger}\left(Q_{u_{i}} m_{d_{j}}^{2}-Q_{d_{j}} m_{u_{i}}^{2}\right)\right)
$$

\subsubsection{Vector-Vector-Vector effective vertices}

The generic effective vertex is

$$
V_{1 \alpha} \overbrace{p_{3}}^{p_{1}} \overbrace{V_{3 \nu}}^{p_{2}}=\frac{i e^{3}}{\pi^{2}} C\left[g_{\alpha \mu}\left(p_{2}-p_{1}\right)_{\nu}+g_{\mu \nu}\left(p_{3}-p_{2}\right)_{\alpha}+g_{\nu \alpha}\left(p_{1}-p_{3}\right)_{\mu}\right]
$$

with the actual values of $V_{1}, V_{2}, V_{3}$ and $C$

$$
\begin{aligned}
& \left.\begin{array}{l}
A A A \\
A A Z \\
A Z Z \\
Z Z Z
\end{array}\right\}: C=0 \\
& A W^{+} W^{-} \quad: \quad C=K \\
& Z W^{+} W^{-} \quad: \quad C=-\frac{c_{w}}{s_{w}} K
\end{aligned}
$$

where

$$
K=\frac{7+4 \lambda_{H V}}{96 s_{w}^{2}}+\frac{1}{48 s_{w}^{2}}\left[\sum_{i=1}^{3} 1+N_{\mathrm{col}} \sum_{i, j=1}^{3}\left(V_{u_{i} d_{j}} V_{d_{j} u_{i}}^{\dagger}\right)\right]
$$

\subsection{Electroweak effective vertices with 4 external legs}

In this section, we give all possible contributing 4-point $\mathrm{R}_{2}$ effective vertices. 


\subsubsection{Scalar-Scalar-Scalar-Scalar effective vertices}

The generic effective vertex is

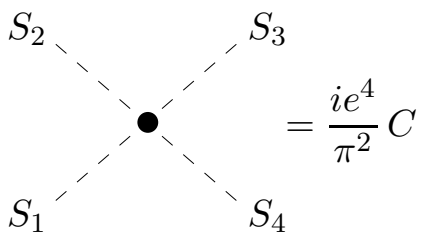

with the actual values of $S_{1}, S_{2}, S_{3}, S_{4}$ and $C$

$$
\begin{aligned}
& \left.\begin{array}{l}
H H H \chi \\
H \chi \chi \chi \\
H \chi \phi^{-} \phi^{+}
\end{array}\right\}: C=0
\end{aligned}
$$

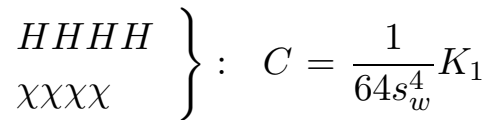

$$
\begin{aligned}
& H H \chi \chi \quad: \quad C=\frac{1}{192 s_{w}^{4}} K_{1} \\
& \left.\begin{array}{l}
H H \phi^{-} \phi^{+} \\
\chi \chi \phi^{-} \phi^{+}
\end{array}\right\}: C=\frac{1}{64 s_{w}^{4}} K_{2} \\
& \phi^{-} \phi^{+} \phi^{-} \phi^{+} \quad: \quad C=\frac{1}{32 s_{w}^{4}} K_{3}
\end{aligned}
$$

where

$$
\begin{aligned}
K_{1}= & \frac{1}{m_{W}^{2}}\left[\frac{5}{m_{W}^{2}} \sum_{i=1}^{6}\left(m_{l_{i}}^{4}+N_{\mathrm{col}} m_{q_{i}}^{4}\right)+\frac{3}{2} m_{H}^{2}\left(1+\frac{1}{2 c_{w}^{2}}\right)\right]+\frac{1-12 \lambda_{H V}}{2}\left(1+\frac{1}{2 c_{w}^{4}}\right) \\
K_{2}= & \frac{1}{m_{W}^{2}}\left[\frac{5}{3 m_{W}^{2}}\left(\sum_{i=1}^{3} m_{e_{i}}^{4}+N_{\mathrm{col}} \sum_{i, j=1}^{3} V_{u_{i} d_{j}} V_{d_{j} u_{i}}^{\dagger}\left(m_{u_{i}}^{4}+m_{d_{j}}^{4}\right)\right)+\frac{1}{2} m_{H}^{2}\left(1+\frac{1}{2 c_{w}^{2}}\right)\right] \\
& +\frac{1-12 \lambda_{H V}}{4}\left(1+\frac{s_{w}^{2}}{3 c_{w}^{2}}\left(1+\frac{1}{c_{w}^{2}}\right)\right) \\
K_{3}= & \frac{1}{m_{W}^{2}}\left[\frac{5}{3 m_{W}^{2}}\left(\sum_{i=1}^{3} m_{e_{i}}^{4}+N_{\mathrm{col}} \sum_{i, j, k, l=1}^{3}\left(V_{u_{i} d_{j}} V_{d_{j} u_{k}}^{\dagger} V_{u_{k} d_{l}} V_{d_{l} u_{i}}^{\dagger}\left(m_{u_{i}}^{2} m_{u_{k}}^{2}+m_{d_{j}}^{2} m_{d_{l}}^{2}\right)\right)\right)\right. \\
& \left.+\frac{1}{2} m_{h}^{2}\left(1+\frac{1}{2 c_{w}^{2}}\right)\right]+\left(\left(\frac{1}{4}-3 \lambda_{H V}\right)\left(1+s_{w}^{4}\right)\right. \\
& \left.+\left(\frac{1}{6}-2 \lambda_{H V}\right)\left(s_{w}^{2}+\frac{2 s_{w}^{6}}{c_{w}^{2}}\right)+\left(\frac{1}{12}-\lambda_{H V}\right) \frac{s_{w}^{8}}{c_{w}^{4}}\right)
\end{aligned}
$$

\subsubsection{Vector-Vector-Vector-Vector effective vertices}

The generic effective vertex is 


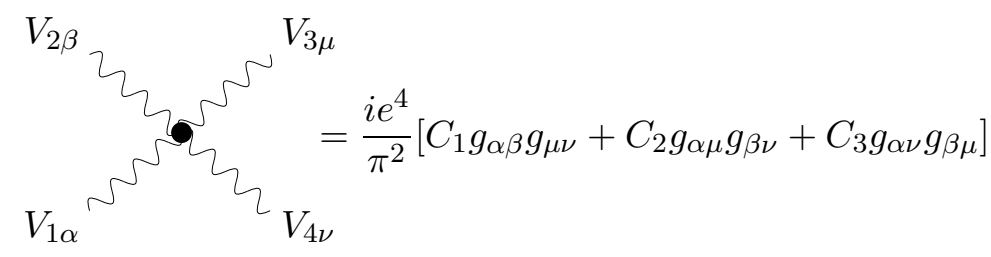

with the actual values of $V_{1}, V_{2}, V_{3}, V_{4} C_{1}, C_{2}$ and $C_{3}$

$$
\begin{aligned}
& A A A A: C_{1}=\frac{1}{12}\left(-1+\sum_{i=1}^{6} Q_{l_{i}}^{4}+N_{\mathrm{col}} \sum_{i=1}^{6} Q_{q_{i}}^{4}\right) \\
& C_{2}=C_{1} \\
& C_{3}=C_{1} \\
& A A A Z: C_{1}=\frac{1}{12}\left[\frac{c_{w}}{s_{w}}+\sum_{i=1}^{6}\left(\frac{s_{w}}{c_{w}} Q_{l_{i}}^{4}-\frac{1}{2 s_{w} c_{w}} Q_{l_{i}}^{3} I_{3 l_{i}}\right)\right. \\
& \left.+N_{\mathrm{col}} \sum_{i=1}^{6}\left(\frac{s_{w}}{c_{w}} Q_{q_{i}}^{4}-\frac{1}{2 s_{w} c_{w}} Q_{q_{i}}^{3} I_{3 q_{i}}\right)\right] \\
& C_{2}=C_{1} \\
& C_{3}=C_{1} \\
& A A Z Z: C_{1}=\frac{1}{12}\left[-\frac{c_{w}^{2}}{s_{w}^{2}}+\frac{1}{2} \sum_{i=1}^{6}\left(\frac{s_{w}^{2}}{c_{w}^{2}} Q_{l_{i}}^{4}+\left(\frac{s_{w}}{c_{w}} Q_{l_{i}}^{2}-\frac{1}{s_{w} c_{w}} Q_{l_{i}} I_{3 l_{i}}\right)^{2}\right)\right. \\
& \left.+\frac{N_{\mathrm{col}}}{2} \sum_{i=1}^{6}\left(\frac{s_{w}^{2}}{c_{w}^{2}} Q_{q_{i}}^{4}+\left(\frac{s_{w}}{c_{w}} Q_{q_{i}}^{2}-\frac{1}{s_{w} c_{w}} Q_{q_{i}} I_{3 q_{i}}\right)^{2}\right)\right] \\
& C_{2}=C_{1} \\
& C_{3}=C_{1} \\
& A Z Z Z: C_{1}=\frac{1}{12}\left[\frac{c_{w}^{3}}{s_{w}^{3}}+\sum_{i=1}^{6}\left(\frac{s_{w}^{3}}{c_{w}^{3}} Q_{l_{i}}^{4}-\frac{3}{2} \frac{s_{w}}{c_{w}^{3}} Q_{l_{i}}^{3} I_{3 l_{i}}\right.\right. \\
& \left.+\frac{3}{2} \frac{1}{s_{w} c_{w}^{3}} Q_{l_{i}}^{2} I_{3 l_{i}}^{2}-\frac{1}{2 s_{w}^{3} c_{w}^{3}} Q_{l_{i}} I_{3 l_{i}}^{3}\right) \\
& +N_{\mathrm{col}} \sum_{i=1}^{6}\left(\frac{s_{w}^{3}}{c_{w}^{3}} Q_{q_{i}}^{4}-\frac{3}{2} \frac{s_{w}}{c_{w}^{3}} Q_{q_{i}}^{3} I_{3 q_{i}}\right. \\
& \left.\left.+\frac{3}{2} \frac{1}{s_{w} c_{w}^{3}} Q_{q_{i}}^{2} I_{3 q_{i}}^{2}-\frac{1}{2 s_{w}^{3} c_{w}^{3}} Q_{q_{i}} I_{3 q_{i}}^{3}\right)\right] \\
& C_{2}=C_{1} \\
& C_{3}=C_{1} \\
& Z Z Z Z: C_{1}=\frac{1}{12}\left[-\frac{c_{w}^{4}}{s_{w}^{4}}+\sum_{i=1}^{6}\left(\frac{s_{w}^{4}}{c_{w}^{4}} Q_{l_{i}}^{4}-2 \frac{s_{w}^{2}}{c_{w}^{4}} Q_{l_{i}}^{3} I_{3 l_{i}}\right.\right. \\
& \left.+\frac{3}{c_{w}^{4}} Q_{l_{i}}^{2} I_{3 l_{i}}^{2}-\frac{2}{s_{w}^{2} c_{w}^{4}} Q_{l_{i}} I_{3 l_{i}}^{3}+\frac{1}{2 s_{w}^{4} c_{w}^{4}} I_{3 l_{i}}^{4}\right)
\end{aligned}
$$




$$
\begin{aligned}
& +N_{\mathrm{col}} \sum_{i=1}^{6}\left(\frac{s_{w}^{4}}{c_{w}^{4}} Q_{q_{i}}^{4}-2 \frac{s_{w}^{2}}{c_{w}^{4}} Q_{q_{i}}^{3} I_{3 q_{i}}\right. \\
& \left.\left.+\frac{3}{c_{w}^{4}} Q_{q_{i}}^{2} I_{3 q_{i}}^{2}-\frac{2}{s_{w}^{2} c_{w}^{4}} Q_{q_{i}} I_{3 q_{i}}^{3}+\frac{1}{2 s_{w}^{4} c_{w}^{4}} I_{3 q_{i}}^{4}\right)\right] \\
& C_{2}=C_{1} \\
& C_{3}=C_{1} \\
& A A W^{-} W^{+} \quad: \quad C_{1}=\frac{1}{16 s_{w}^{2}}\left[\frac{10+4 \lambda_{H V}}{3}+\sum_{i=1}^{3} 1+\frac{25}{27} N_{\mathrm{col}} \sum_{i, j=1}^{3}\left(V_{u_{i} d_{j}} V_{d_{j} u_{i}}^{\dagger}\right)\right] \\
& C_{2}=-\frac{1}{16 s_{w}^{2}}\left[\frac{7+2 \lambda_{H V}}{3}+\frac{1}{3} \sum_{i=1}^{3} 1+\frac{11}{27} N_{\mathrm{col}} \sum_{i, j=1}^{3}\left(V_{u_{i} d_{j}} V_{d_{j} u_{i}}^{\dagger}\right)\right] \\
& C_{3}=C_{2} \\
& A Z W^{-} W^{+}: C_{1}=\frac{1}{16 s_{w} c_{w}}\left[-\frac{\left(10+4 \lambda_{H V}\right) c_{w}^{2}}{3 s_{w}^{2}}+\left(1-\frac{11}{12 s_{w}^{2}}\right) \sum_{i=1}^{3} 1\right. \\
& \left.+N_{\mathrm{col}} \sum_{i, j=1}^{3}\left(V_{u_{i} d_{j}} V_{d_{j} u_{i}}^{\dagger}\left(\frac{25}{27}-\frac{11}{12 s_{w}^{2}}\right)\right)\right] \\
& C_{2}=\frac{1}{16 s_{w} c_{w}}\left[\frac{7+2 \lambda_{H V}}{3} \frac{c_{w}^{2}}{s_{w}^{2}}+\left(\frac{5}{12 s_{w}^{2}}-\frac{1}{3}\right) \sum_{i=1}^{3} 1\right. \\
& \left.+N_{\mathrm{col}} \sum_{i, j=1}^{3}\left(V_{u_{i} d_{j}} V_{d_{j} u_{i}}^{\dagger}\left(\frac{5}{12 s_{w}^{2}}-\frac{11}{27}\right)\right)\right] \\
& C_{3}=C_{2} \\
& Z Z W^{-} W^{+}: C_{1}=\frac{\left(5+2 \lambda_{H V}\right) c_{w}^{2}}{24 s_{w}^{4}}+\frac{1}{16 c_{w}^{2}}\left[\left(1-\frac{11}{6 s_{w}^{2}}+\frac{11}{12 s_{w}^{4}}\right) \sum_{i=1}^{3} 1\right. \\
& \left.+N_{\text {col }}\left(\frac{25}{27}-\frac{11}{6 s_{w}^{2}}+\frac{11}{12 s_{w}^{4}}\right) \sum_{i, j=1}^{3}\left(V_{u_{i} d_{j}} V_{d_{j} u_{i}}^{\dagger}\right)\right] \\
& C_{2}=-\frac{\left(7+2 \lambda_{H V}\right) c_{w}^{2}}{48 s_{w}^{4}}+\frac{1}{16 c_{w}^{2}}\left[\left(-\frac{1}{3}+\frac{5}{6 s_{w}^{2}}-\frac{5}{12 s_{w}^{4}}\right) \sum_{i=1}^{3} 1\right. \\
& \left.+N_{\mathrm{col}}\left(-\frac{11}{27}+\frac{5}{6 s_{w}^{2}}-\frac{5}{12 s_{w}^{4}}\right) \sum_{i, j=1}^{3}\left(V_{u_{i} d_{j}} V_{d_{j} u_{i}}^{\dagger}\right)\right] \\
& C_{3}=C_{2}
\end{aligned}
$$




$$
\begin{aligned}
W^{-} W^{+} W^{-} W^{+}: C_{1}= & \frac{1}{16 s_{w}^{4}}\left[\frac{3+2 \lambda_{H V}}{3}+\frac{1}{2} \sum_{i=1}^{3} 1\right. \\
& \left.+\frac{N_{\mathrm{col}}}{2} \sum_{i, j, k, m=1}^{3}\left(V_{u_{i} d_{j}} V_{d_{j} u_{k}}^{\dagger} V_{u_{k} d_{m}} V_{d_{m} u_{i}}^{\dagger}\right)\right] \\
C_{2}= & -\frac{1}{8 s_{w}^{4}}\left[\frac{7+2 \lambda_{H V}}{3}+\frac{5}{12} \sum_{i=1}^{3} 1\right. \\
& \left.+\frac{5}{12} N_{\mathrm{col}} \sum_{i, j, k, m=1}^{3}\left(V_{u_{i} d_{j}} V_{d_{j} u_{k}}^{\dagger} V_{u_{k} d_{m}} V_{d_{m} u_{i}}^{\dagger}\right)\right] \\
C_{3}= & C_{1}
\end{aligned}
$$

\subsubsection{Scalar-Scalar-Vector-Vector effective vertices}

The generic effective vertex is

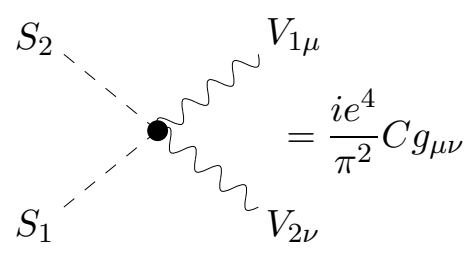

with the actual values of $S_{1}, S_{2}, V_{1}, V_{2}$ and $C$

$$
\begin{aligned}
& \left.\begin{array}{l}
H \chi A A \\
H \chi A Z \\
H \chi Z Z \\
H \chi W^{+} W^{-}
\end{array}\right\}: C=0 \\
& \left.\begin{array}{l}
\text { HHAA } \\
\chi \chi A A
\end{array}\right\}: \quad C=\frac{1}{16 s_{w}^{2}}\left\{\frac{1}{12}-\frac{1}{m_{W}^{2}}\left[\sum_{i=1}^{6}\left(Q_{l_{i}}^{2} m_{l_{i}}^{2}\right)+N_{\mathrm{col}} \sum_{i=1}^{6}\left(Q_{q_{i}}^{2} m_{q_{i}}^{2}\right)\right]\right\} \\
& \left.\begin{array}{l}
H H A Z \\
\chi \chi A Z
\end{array}\right\}: \quad C=\frac{1}{16 s_{w}}\left\{\frac{1-6 s_{w} c_{w}+7 s_{w}^{2}}{12 s_{w}^{2} c_{w}}+\frac{1}{m_{W}^{2} c_{w}}\left[\sum_{i=1}^{6}\left(Q_{l_{i}} m_{l_{i}}^{2}\left(\frac{I_{3 l_{i}}}{2 s_{w}^{2}}-Q_{l_{i}}\right)\right)\right.\right. \\
& \left.\left.+N_{\text {col }} \sum_{i=1}^{6}\left(Q_{q_{i}} m_{q_{i}}^{2}\left(\frac{I_{3 q_{i}}}{2 s_{w}^{2}}-Q_{q_{i}}\right)\right)\right]\right\}
\end{aligned}
$$

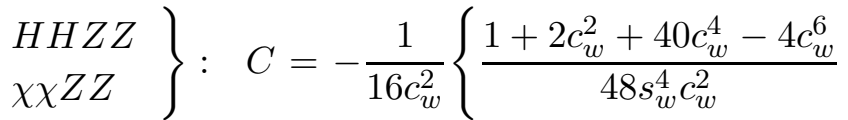

$$
\begin{aligned}
& +\frac{1}{m_{W}^{2}}\left[\sum_{i=1}^{6}\left(m_{l_{i}}^{2}\left(Q_{l_{i}}^{2}+\frac{4 I_{3 l_{i}}^{2}}{3 s_{w}^{4}}-\frac{Q_{l_{i}} I_{3 l_{i}}}{s_{w}^{2}}\right)\right)\right. \\
& \left.\left.+N_{\mathrm{col}} \sum_{i=1}^{6}\left(m_{q_{i}}^{2}\left(Q_{q_{i}}^{2}+\frac{4 I_{3 q_{i}}^{2}}{3 s_{w}^{4}}-\frac{Q_{q_{i}} I_{3 q_{i}}}{s_{w}^{2}}\right)\right)\right]\right\}
\end{aligned}
$$




$$
\begin{aligned}
& \left.\begin{array}{l}
H H W^{-} W^{+} \\
\chi \chi W^{-} W^{+}
\end{array}\right\}: C=-\frac{1}{48 s_{w}^{4}}\left\{\frac{1+38 c_{w}^{2}}{16 c_{w}^{2}}\right. \\
& \left.+\frac{1}{m_{W}^{2}}\left[\sum_{i=1}^{3} m_{e_{i}}^{2}+N_{\mathrm{col}} \sum_{i, j=1}^{3}\left(V_{u_{i} d_{j}} V_{d_{j} u_{i}}^{\dagger}\left(m_{u_{i}}^{2}+m_{d_{j}}^{2}\right)\right)\right]\right\} \\
& \left.\begin{array}{l}
H \phi^{+} W^{-} A \\
\phi^{-} H A W^{+} \\
\chi \phi^{+} W^{-} A
\end{array}\right\}: C=K_{1} \\
& \phi^{-} \chi A W^{+}: C=-K_{1} \\
& \left.\begin{array}{l}
H \phi^{+} W^{-} Z \\
\phi^{-} H Z W^{+} \\
\chi \phi^{+} W^{-} Z
\end{array}\right\}: C=K_{2} \\
& \phi^{-} \chi Z W^{+}: C=-K_{2} \\
& \phi^{-} \phi^{+} A A \quad: \quad C=-\frac{1}{12 s_{w}^{2}}\left\{\frac{1+21 c_{w}^{2}}{16 c_{w}^{2}}+\frac{1}{m_{W}^{2}}\left[\sum_{i=1}^{3} m_{e_{i}}^{2}\right.\right. \\
& \left.\left.+\frac{5}{6} N_{\mathrm{col}} \sum_{i, j=1}^{3}\left(V_{u_{i} d_{j}} V_{d_{j} u_{i}}^{\dagger}\left(m_{u_{i}}^{2}+m_{d_{j}}^{2}\right)\right)\right]\right\} \\
& \phi^{-} \phi^{+} A Z \quad: \quad C=\frac{1}{12 s_{w} c_{w}}\left\{\frac{1-3 s_{w} c_{w}-8 c_{w}^{2}+21 c_{w}^{4}}{16 s_{w}^{2} c_{w}^{2}}\right. \\
& -\frac{1}{m_{W}^{2}}\left[\sum_{i=1}^{3}\left(m_{e_{i}}^{2} Q_{e_{i}}\left(Q_{e_{i}}+\frac{5}{8} \frac{I_{3 \nu_{i}}}{s_{w}^{2}}\right)\right)\right. \\
& +N_{\mathrm{col}} \sum_{i, j=1}^{3}\left[V _ { u _ { i } d _ { j } } V _ { d _ { j } u _ { i } } ^ { \dagger } \left(m_{u_{i}}^{2}\left(\frac{5}{6}-\frac{I_{3 d_{i}}}{s_{w}^{2}}\left(Q_{d_{j}}-\frac{5}{8} Q_{u_{i}}\right)\right)\right.\right. \\
& \left.\left.\left.\left.+m_{d_{j}}^{2}\left(\frac{5}{6}-\frac{I_{3 u_{i}}}{s_{w}^{2}}\left(Q_{u_{i}}-\frac{5}{8} Q_{d_{j}}\right)\right)\right)\right]\right]\right\} \\
& \phi^{-} \phi^{+} Z Z \quad: \quad C=\frac{1}{12 c_{w}^{2}}\left\{\frac{-1+2 c_{w}^{2}+44 c_{w}^{4}-84 c_{w}^{6}}{64 s_{w}^{4} c_{w}^{2}}\right. \\
& -\frac{1}{m_{W}^{2}}\left[\sum_{i=1}^{3}\left(m_{e_{i}}^{2}\left(Q_{e_{i}}^{2}+\frac{5}{4} \frac{Q_{e_{i}} I_{3 \nu_{i}}}{s_{w}^{2}}+\frac{I_{3 \nu_{i}}^{2}}{s_{w}^{4}}\right)\right)\right. \\
& +N_{\mathrm{col}} \sum_{i, j=1}^{3}\left[V _ { u _ { i } d _ { j } } V _ { d _ { j } u _ { i } } ^ { \dagger } \left(m_{u_{i}}^{2}\left(\frac{5}{6}-\frac{I_{3 d_{i}}}{s_{w}^{2}}\left(2 Q_{d_{j}}-\frac{5}{4} Q_{u_{i}}\right)+\frac{I_{3 d_{i}}^{2}}{s_{w}^{4}}\right)\right.\right. \\
& \left.\left.\left.\left.+m_{d_{j}}^{2}\left(\frac{5}{6}-\frac{I_{3 u_{i}}}{s_{w}^{2}}\left(2 Q_{u_{i}}-\frac{5}{4} Q_{d_{j}}\right)\right)+\frac{I_{3 u_{i}}^{2}}{s_{w}^{4}}\right)\right]\right]\right\}
\end{aligned}
$$




$$
\begin{aligned}
\phi^{-} \phi^{+} W^{-} W^{+}: C= & -\frac{1}{48 s_{w}^{4}}\left\{\frac { 1 } { m _ { W } ^ { 2 } } \left[\left(\sum_{i=1}^{3} m_{e_{i}}^{2}\right.\right.\right. \\
& \left.\left.+N_{\mathrm{col}} \sum_{i, j, k, l=1}^{3}\left(V_{u_{i} d_{j}} V_{d_{j} u_{k}}^{\dagger} V_{u_{k} d_{l}} V_{d_{l} u_{i}}^{\dagger}\left(m_{u_{i}} m_{u_{k}}+m_{d_{j}} m_{d_{l}}\right)\right)\right)\right] \\
& \left.+\frac{192 c_{w}^{6}-264 c_{w}^{4}-192 s_{w}^{3} c_{w}^{3}+116 c_{w}^{2}+1}{16 c_{w}^{2}}\right\}
\end{aligned}
$$

with

$$
\begin{aligned}
K_{1} & =\frac{1}{24 s_{w}^{3}}\left\{\frac{1+34 c_{w}^{2}-24 c_{w}^{3} s_{w}-24 c_{w}^{4}}{32 c_{w}^{2}}+K\right\} \\
K_{2} & =\frac{1}{24 s_{w}^{2} c_{w}}\left\{\frac{1+10 c_{w}^{2}+24 c_{w}^{3} s_{w}+24 c_{w}^{4}}{32 c_{w}^{2}}+K\right\} \\
K & =\frac{1}{8 m_{W}^{2}}\left[\sum_{i=1}^{3} m_{e_{i}}^{2}+N_{\mathrm{col}} \sum_{i, j=1}^{3}\left(V_{u_{i} d_{j}} V_{d_{j} u_{i}}^{\dagger}\left(3 m_{d_{j}}^{2}+2 m_{u_{i}}^{2}\right)\right)\right]
\end{aligned}
$$

\subsection{Mixed Electroweak/QCD corrections}

In [25], all mixed $\mathrm{R}_{2} \mathrm{QCD} /$ Electroweak vertices with internal QCD particle and external weak fields are presented. For completeness, we give here the only contributing Mixed Electroweak/QCD $\mathrm{R}_{2}$ effective vertex, with internal EW particles and external colored states.

\subsubsection{Gluon-Quark-Quark effective vertex}

The generic effective vertex is

$$
G_{\mu}^{a}+000<\bar{Q}_{k}^{Q_{l}}=\frac{i g_{s} e^{2}}{\pi^{2}} t_{k l}^{a}\left(C_{-} \Omega^{-}+C_{+} \Omega^{+}\right) \gamma_{\mu}
$$

with the actual values of $Q, \bar{Q}, C_{-}$and $C_{+}$

$$
\begin{aligned}
u \bar{u}: C_{-}= & \frac{1}{8}\left[\left(1+4 \lambda_{H V}\right) \frac{Q_{u}^{2}}{c_{w}^{2}}+\frac{\left(1+2 \lambda_{H V}\right) m_{u}^{2}}{2 s_{w}^{2} m_{W}^{2}}\left(\frac{1}{2} \sum_{j=1}^{3}\left(V_{u d_{j}} V_{d_{j} u}^{\dagger}\right)+\frac{1}{4}+I_{3 u}^{2}\right)\right] \\
C_{+}= & \frac{1}{8}\left[\frac{\left(1+4 \lambda_{H V}\right)}{c_{w}^{2}}\left(Q_{u}^{2}+\frac{I_{3 u}^{2}}{s_{w}^{2}}-2 Q_{u} I_{3 u}\right)+\frac{\left(1+4 \lambda_{H V}\right)}{2 s_{w}^{2}} \sum_{j=1}^{3}\left(V_{u d_{j}} V_{d_{j} u}^{\dagger}\right)\right. \\
& \left.+\frac{\left(1+2 \lambda_{H V}\right)}{2 m_{W}^{2} s_{w}^{2}}\left(\frac{1}{2} \sum_{j=1}^{3}\left(V_{u d_{j}} V_{d_{j} u}^{\dagger} m_{d_{j}}^{2}\right)+m_{u}^{2}\left(\frac{1}{4}+I_{3 u}^{2}\right)\right)\right] \\
d \bar{d}: C_{-}= & \frac{1}{8}\left[\left(1+4 \lambda_{H V}\right) \frac{Q_{d}^{2}}{c_{w}^{2}}+\frac{\left(1+2 \lambda_{H V}\right) m_{d}^{2}}{2 s_{w}^{2} m_{W}^{2}}\left(\frac{1}{2} \sum_{i=1}^{3}\left(V_{u_{i} d} V_{d u_{i}}^{\dagger}\right)+\frac{1}{4}+I_{3 d}^{2}\right)\right]
\end{aligned}
$$




$$
\begin{aligned}
C_{+}= & \frac{1}{8}\left[\frac{\left(1+4 \lambda_{H V}\right)}{c_{w}^{2}}\left(Q_{d}^{2}+\frac{I_{3 d}^{2}}{s_{w}^{2}}-2 Q_{d} I_{3 d}\right)+\frac{\left(1+4 \lambda_{H V}\right)}{2 s_{w}^{2}} \sum_{i=1}^{3}\left(V_{u_{i} d} V_{d u_{i}}^{\dagger}\right)\right. \\
& \left.+\frac{\left(1+2 \lambda_{H V}\right)}{2 m_{W}^{2} s_{w}^{2}}\left(\frac{1}{2} \sum_{j=1}^{3}\left(V_{u_{i} d} V_{d u_{i}}^{\dagger} m_{u_{i}}^{2}\right)+m_{d}^{2}\left(\frac{1}{4}+I_{3 d}^{2}\right)\right)\right]
\end{aligned}
$$

\section{Tests and findings}

We performed several checks on our formulae. First of all, we derived them by means of two independent calculations, performed with the help of FORM [33, 34] and FeynArts [36], secondly, we explicitly checked the gauge invariance of our results with the help of the Ward Identities listed in appendix A, that we derived, by using the Background Field Method described in [37], in the way we detail in the appendix. Given the fact that only $\mathrm{R}=\mathrm{R}_{1}+\mathrm{R}_{2}$ is gauge invariant, we adopted the following strategy. The terms proportional to $\lambda_{H V}$ in our effective vertices are expected to be gauge invariant by themselves. Such terms can only be generated by $\mathrm{R}_{2}$, so that we could explicitly check, by using FORM, that this part of our results fulfills all of the Ward identities of appendix A, both in the 't Hooft-Feynman gauge and in the Background Field Method approach. This provides an explicit test of the gauge invariance of the Four Dimensional Helicity regularization scheme in the complete Standard Model at 1-loop, and we consider this result as a by-product of our calculation.

To also test the parts not proportional to $\lambda_{H V}$, we computed analytically $\mathrm{R}_{1},{ }^{4}$ we added it to $R_{2}$ and checked that the quantity $R_{1}+R_{2}$ fulfills all of the 2-point and 3-point Ward identities listed in the appendix. In the 4-point case, many new vertices are present in $\mathrm{R}_{1}$ that do not contribute to $\mathrm{R}_{2}$, such as VVVS, and, given the fact that, after all, we just need to check $R_{2}$, we limited ourselves to verify the first six 4-point Ward identities given in appendix A.6, which are the only ones including both the VVVV and VVV vertices, but not VVVS. The described gauge invariance test on $R_{1}+R_{2}$ is a very powerful and non trivial one. In fact, the analytic expressions for $\mathrm{R}_{1}$ are, in general, much more complicated than the ones for $\mathrm{R}_{2}$, involving a huge amount of terms with different combinations/powers of Gram determinants.

\section{Conclusions}

In the last few years, new techniques have been developed to efficiently deal with the problem of computing the radiative corrections needed to cope with the complicated phenomenology expected at LHC and ILC. Nowadays, thanks to the OPP technique, the so called Cut Constructible part of the virtual 1-loop amplitudes can be obtained, in a purely numerical way, by means of a calculation of the same conceptual complexity of a tree level one. However, the determination of the remaining rational part $\mathrm{R}$ of the amplitude requires a different strategy. In the treatment at the integrand level, that we follow in this

\footnotetext{
${ }^{4}$ We extracted the $\mathrm{R}_{1}$ part of the contributing tensor integrals by using the Passarino-Veltman [38] reduction technique and by further checking numerically the expressions with the help of CutTools [30].
} 
paper, a piece of $\mathrm{R}$, called $\mathrm{R}_{1}$, can be directly linked to the Cut Constructible part of the amplitude, and it is therefore numerically and automatically produced, in the OPP framework, by codes like CutTools. The remaining part of $\mathrm{R}$, called $\mathrm{R}_{2}$, cannot be determined numerically in 4 dimensions, and requires an explicit computation in terms of the vertices of the theory at hand, up to four external legs. From the knowledge of these vertices, a finite set of effective tree level Feynman rules can be extracted to be used to compute $\mathrm{R}_{2}$ for processes with an arbitrary number of external legs. Such effective $\mathrm{R}_{2}$ Feynman rules have been already given, in the literature, for QED and QCD and, in this paper, we completed the list by computing and presenting the set of $\mathrm{R}_{2}$ Feynman rules for the Electroweak sector, which was the last missing piece for completely automatizing, in the framework of the OPP method, the 1-loop calculations in the $\mathrm{SU}(3) \times \mathrm{SU}(2) \times \mathrm{U}(1)$ Standard Model.

In addition, since $R_{2}$ is the only part of the amplitude sensitive to the choice of the regularization scheme, we explicitly proved, by checking a large set of Ward identities, the gauge invariance of the Four Dimensional Helicity regularization scheme in the full Electroweak sector at 1-loop.

\section{Acknowledgments}

We would like to thank Costas Papadopoulos, Ronald Kleiss and Stefan Dittmaier for useful discussions. R.P. is also indebted with Mauro Moretti and Fulvio Piccinini for discussing the gauge invariance arguments presented in section 2. R.P.'s and I.M.'s research was partially supported by the RTN European Programme MRTN-CT-2006-035505 (HEPTOOLS, Tools and Precision Calculations for Physics Discoveries at Colliders). M.V.G.'s research was supported by INFN. The research of R.P. and M.V.G. was also supported by the MEC project FPA2008-02984.

\section{A Ward identities}

The Background Field Method (BFM) is a technique for quantizing gauge theories without losing explicit gauge invariance of the effective action [37, 39-42]. Starting from a classical Lagrangian, one can achieve this by decomposing the usual fields into background fields and quantum fields. Then, the background fields are treated as external sources, while the quantum fields are variables of integration in the functional integral. A gauge fixing term is added, which only breaks the invariance with respect to the quantum gauge transformations, while the invariance with respect to background-field gauge transformations is preserved. From the Lagrangian mentioned above, one can construct an effective action $\Gamma[\hat{V}, \hat{S}, F, \bar{F}]$, where $\hat{V}$ refers to the background gauge fields, $\hat{S}$ to the background scalar fields and $F, \bar{F}$ to the fermion fields (for all fields that do not enter the gauge-fixing term, quantization is identical in the BFM and in the conventional formalism. Their Feynman rules for the background fields and quantum fields are also identical, so there is no need to distinguish them). This effective action is invariant under the background gauge transformations given in eqs. 21, 22 of [37]. This invariance implies that

$$
\frac{\delta \Gamma}{\delta \hat{\theta}^{a}}=0
$$


where $a=A, Z, W^{ \pm}$and $\hat{\theta}^{a}$ are the infinitesimal gauge transformations of the background fields. By combining these formulas with eqs. 21, 22 of [37], one can produce eqs. 4, 5 and 6 of [41]. By differentiating them with respect to background fields and setting the fields equal to zero, one obtains Ward identities for the vertex functions that are precisely the Ward identities related to the classical Lagrangian. In the papers [37] and [41] some of these Ward identities are listed (see also [43]). In the following, we extend this list by producing more Ward identities useful for our checks. ${ }^{5}$

\section{A.1 Ward identities involving VV, VS and SS}

$$
\begin{aligned}
k^{\mu} \Gamma_{\mu \nu}^{A A}(k,-k)=k^{\mu} \Gamma_{\mu \nu}^{A Z}(k,-k) & =0 \\
k^{\mu} \Gamma_{\mu \nu}^{Z Z}(k,-k)-i M_{Z} \Gamma_{\nu}^{\chi Z}(k,-k) & =0 \\
k^{\mu} \Gamma_{\mu \nu}^{W^{ \pm} W^{\mp}}(k,-k) \mp M_{W} \Gamma_{\nu}^{\phi^{ \pm} W^{\mp}}(k,-k) & =0 \\
k^{\mu} \Gamma_{\mu \nu}^{Z \chi}(k,-k)-i M_{Z} \Gamma_{\nu}^{\chi \chi}(k,-k)+\frac{i e}{2 c_{w} s_{w}} T^{H} & =0 \\
k^{\mu} \Gamma_{\mu \nu}^{W^{ \pm} \phi^{\mp}}(k,-k) \mp M_{W} \Gamma_{\nu}^{\phi^{ \pm} \phi^{\mp}}(k,-k) \pm \frac{e}{2 s_{w}} T^{H} & =0
\end{aligned}
$$

In the previous identities, $T^{H}$ is the Higgs tadpole contribution. We have found a nonvanishing $\mathrm{R}_{2}$ contribution to $T^{H}$, due to the coupling of $H$ with $Z$ and $W$, while $\mathrm{R}_{1}$ does not contribute to $T^{H}$.

\section{A.2 Ward identities involving VFF, SFF and FF}

$$
\begin{aligned}
& k^{\mu} \Gamma_{\mu}^{A \bar{f} f}(k, \bar{p}, p)+e Q_{f}\left(\Gamma^{\bar{f} f}(\bar{p}, k+p)-\Gamma^{\bar{f} f}(k+\bar{p}, p)\right)=0 \\
& k^{\mu} \Gamma_{\mu}^{Z \bar{f} f}(k, \bar{p}, p)-i M_{Z} \Gamma^{\chi \bar{f} f}(k, \bar{p}, p)-e\left(\Gamma^{\bar{f} f}(\bar{p}, k+p)\left(v_{f}-a_{f} \gamma_{5}\right)\right. \\
& \left.-\left(v_{f}+a_{f} \gamma_{5}\right) \Gamma^{\bar{f} f}(k+\bar{p}, p)\right)=0 \\
& k^{\mu} \Gamma_{\mu}^{W^{+} \bar{f}_{u} f_{d}}(k, \bar{p}, p)-M_{W} \Gamma^{\phi^{+} \bar{f}_{u} f_{d}}(k, \bar{p}, p)-\frac{e}{\sqrt{2} s_{w}}\left(\Gamma^{\bar{f}_{u} f_{u}}(\bar{p}, k+p) \Omega_{-}\right. \\
& \left.-\Omega_{+} \Gamma^{\bar{f}_{d} f_{d}}(k+\bar{p}, p)\right)=0 \\
& k^{\mu} \Gamma_{\mu}^{W^{-} \bar{f}_{d} f_{u}}(k, \bar{p}, p)+M_{W} \Gamma^{\phi^{-} \bar{f}_{d} f_{u}}(k, \bar{p}, p)-\frac{e}{\sqrt{2} s_{w}}\left(\Gamma^{\bar{f}_{d} f_{d}}(\bar{p}, k+p) \Omega_{-}\right. \\
& \left.-\Omega_{+} \Gamma^{\bar{f}_{u} f_{u}}(k+\bar{p}, p)\right)=0
\end{aligned}
$$

In the previous expressions, $f_{u}$ is a fermion with $I_{3 f}=1 / 2, f_{d}$ is the fermion of the same weak-isospin doublet with $I_{3 f}=-1 / 2, v_{f}=\left(I_{3 f}-2 s_{w}^{2} Q_{f}\right) /\left(2 s_{w} c_{w}\right)$ and $a_{f}=I_{3 f} /\left(2 s_{w} c_{w}\right)$.

\section{A.3 Ward identities involving VVV, VVS and VV}

$$
\begin{aligned}
k^{\mu} \Gamma_{\mu \nu \sigma}^{A W^{+} W^{-}}\left(k, k_{+}, k_{-}\right)-e\left(\Gamma_{\nu \sigma}^{W^{+} W^{-}}\left(k_{+}, k+k_{-}\right)-\Gamma_{\nu \sigma}^{W^{+} W^{-}}\left(k+k_{+}, k_{-}\right)\right) & =0 \\
k_{+}^{\mu} \Gamma_{\mu \nu \sigma}^{W^{+} W^{-} A}\left(k_{+}, k_{-}, k\right)-M_{W} \Gamma_{\nu \sigma}^{\phi^{+} W^{-}} A\left(k_{+}, k_{-}, k\right)-e \Gamma_{\sigma \nu}^{W^{+} W^{-}}\left(k+k_{+}, k_{-}\right) & \\
+e\left(\Gamma_{\sigma \nu}^{A A}\left(k, k_{+}+k_{-}\right)-\frac{c_{w}}{s_{w}} \Gamma_{\sigma \nu}^{A Z}\left(k, k_{+}+k_{-}\right)\right) & =0
\end{aligned}
$$

\footnotetext{
${ }^{5}$ We assume $V_{u d}=V_{d u}^{\dagger}=1$ and understand a sum over colors.
} 


$$
\begin{array}{r}
k_{-}^{\mu} \Gamma_{\mu \nu \sigma}^{W^{-} W^{+} A}\left(k_{-}, k_{+}, k\right)+M_{W} \Gamma_{\nu \sigma}^{\phi^{-} W^{+} A}\left(k_{-}, k_{+}, k\right)+e \Gamma_{\sigma \nu}^{W^{-} W^{+}}\left(k+k_{-}, k_{+}\right) \\
-e\left(\Gamma_{\sigma \nu}^{A A}\left(k, k_{+}+k_{-}\right)-\frac{c_{w}}{s_{w}} \Gamma_{\sigma \nu}^{A Z}\left(k, k_{+}+k_{-}\right)\right)=0 \\
k^{\mu} \Gamma_{\mu \nu \sigma}^{Z W^{+} W^{-}}\left(k, k_{+}, k_{-}\right)-i M_{Z} \Gamma_{\nu \sigma}^{\chi W^{+} W^{-}}\left(k, k_{+}, k_{-}\right)-e \frac{c_{w}}{s_{w}}\left(\Gamma_{\nu \sigma}^{W^{+} W^{-}}\left(k+k_{+}, k_{-}\right)\right. \\
\left.-\Gamma_{\sigma \nu}^{W-W^{+}}\left(k+k_{-}, k_{+}\right)\right)=0 \\
k_{+}^{\mu} \Gamma_{\mu \nu \sigma}^{W^{+} W^{-} Z}\left(k_{+}, k_{-}, k\right)-M_{W} \Gamma_{\nu \sigma}^{\phi^{+} W^{-} Z}\left(k_{+}, k_{-}, k\right)+e \frac{c_{w}}{s_{w}} \Gamma_{\sigma \nu}^{W^{+} W^{-}}\left(k+k_{+}, k_{-}\right) \\
+e\left(\Gamma_{\nu \sigma}^{A Z}\left(k_{+}+k_{-}, k\right)-\frac{c_{w}}{s_{w}} \Gamma_{\nu \sigma}^{Z Z}\left(k_{+}+k_{-}, k\right)\right)=0 \\
k_{-}^{\mu} \Gamma_{\mu \nu \sigma}^{W^{-} W^{+} Z}\left(k_{-}, k_{+}, k\right)+M_{W} \Gamma_{\nu \sigma}^{\phi^{-} W^{+} Z}\left(k_{-}, k_{+}, k\right)-e \frac{c_{w}}{s_{w}} \Gamma_{\sigma \nu}^{W} W^{+}\left(k+k_{-}, k_{+}\right) \\
-e\left(\Gamma_{\nu \sigma}^{A Z}\left(k_{+}+k_{-}, k\right)-\frac{c_{w}}{s_{w}} \Gamma_{\nu \sigma}^{Z Z}\left(k_{+}+k_{-}, k\right)\right)=0
\end{array}
$$

\section{A.4 Ward identities involving VVS, VSS and VS}

$$
\begin{aligned}
& k_{1}^{\mu} \Gamma_{\mu \nu}^{A A H}\left(k_{1}, k_{2}, k_{3}\right)=k_{1}^{\mu} \Gamma_{\mu \nu}^{A A \chi}\left(k_{1}, k_{2}, k_{3}\right) \\
& =k_{1}^{\mu} \Gamma_{\mu \nu}^{A Z H}\left(k_{1}, k_{2}, k_{3}\right)=k_{1}^{\mu} \Gamma_{\mu \nu}^{A Z \chi}\left(k_{1}, k_{2}, k_{3}\right)=0 \\
& k^{\mu} \Gamma_{\mu \nu}^{A W^{+} \phi^{-}}\left(k, k_{+}, k_{-}\right)+e \Gamma_{\nu}^{W^{+} \phi^{-}}\left(k+k_{+}, k_{-}\right)-e \Gamma_{\nu}^{\phi^{-} W^{+}}\left(k+k_{-}, k_{+}\right)=0 \\
& k^{\mu} \Gamma_{\mu \nu}^{A W^{-} \phi^{+}}\left(k, k_{-}, k_{+}\right)-e \Gamma_{\nu}^{W^{-} \phi^{+}}\left(k+k_{-}, k_{+}\right)+e \Gamma_{\nu}^{\phi^{+} W^{-}}\left(k+k_{+}, k_{-}\right)=0 \\
& k_{1}^{\mu} \Gamma_{\mu \nu}^{Z A H}\left(k_{1}, k_{2}, k_{3}\right)-i M_{Z} \Gamma_{\nu}^{\chi A H}\left(k_{1}, k_{2}, k_{3}\right)-\frac{i e}{2 c_{w} s_{w}} \Gamma_{\nu}^{\chi A}\left(k_{1}+k_{3}, k_{2}\right)=0 \\
& k_{1}^{\mu} \Gamma_{\mu \nu}^{Z A \chi}\left(k_{1}, k_{2}, k_{3}\right)-i M_{Z} \Gamma_{\nu}^{\chi A \chi}\left(k_{1}, k_{2}, k_{3}\right)+\frac{i e}{2 c_{w} s_{w}} \Gamma_{\nu}^{H A}\left(k_{1}+k_{3}, k_{2}\right)=0 \\
& k_{1}^{\mu} \Gamma_{\mu \nu}^{Z Z H}\left(k_{1}, k_{2}, k_{3}\right)-i M_{Z} \Gamma_{\nu}^{\chi Z H}\left(k_{1}, k_{2}, k_{3}\right)-\frac{i e}{2 c_{w} s_{w}} \Gamma_{\nu}^{\chi Z}\left(k_{1}+k_{3}, k_{2}\right)=0 \\
& k_{1}^{\mu} \Gamma_{\mu \nu}^{Z Z \chi}\left(k_{1}, k_{2}, k_{3}\right)-i M_{Z} \Gamma_{\nu}^{\chi Z \chi}\left(k_{1}, k_{2}, k_{3}\right)+\frac{i e}{2 c_{w} s_{w}} \Gamma_{\nu}^{H Z}\left(k_{1}+k_{3}, k_{2}\right)=0 \\
& k^{\mu} \Gamma_{\mu \nu}^{Z W^{+} \phi^{-}}\left(k, k_{+}, k_{-}\right)-i M_{Z} \Gamma_{\nu}^{\chi W^{+} \phi^{-}}\left(k, k_{+}, k_{-}\right) \\
& -e \frac{c_{w}}{s_{w}} \Gamma_{\nu}^{W^{+} \phi^{-}}\left(k+k_{+}, k_{-}\right)+e \frac{c_{w}^{2}-s_{w}^{2}}{2 c_{w} s_{w}} \Gamma_{\nu}^{\phi^{-} W^{+}}\left(k+k_{-}, k_{+}\right)=0 \\
& k^{\mu} \Gamma_{\mu \nu}^{Z W^{-} \phi^{+}}\left(k, k_{-}, k_{+}\right)-i M_{Z} \Gamma_{\nu}^{\chi W^{-} \phi^{+}}\left(k, k_{-}, k_{+}\right) \\
& +e \frac{c_{w}}{s_{w}} \Gamma_{\nu}^{W^{-} \phi^{+}}\left(k+k_{-}, k_{+}\right)-e \frac{c_{w}^{2}-s_{w}^{2}}{2 c_{w} s_{w}} \Gamma_{\nu}^{\phi^{+} W^{-}}\left(k+k_{+}, k_{-}\right)=0 \\
& k_{+}^{\mu} \Gamma_{\mu \nu}^{W^{+} A \phi^{-}}\left(k_{+}, k, k_{-}\right)-M_{W} \Gamma_{\nu}^{\phi^{+} A \phi^{-}}\left(k_{+}, k, k_{-}\right) \\
& -e \Gamma_{\nu}^{W^{+} \phi^{-}}\left(k+k_{+}, k_{-}\right)+\frac{e}{2 s_{w}}\left(\Gamma_{\nu}^{H A}\left(k_{+}+k_{-}, k\right)+i \Gamma_{\nu}^{\chi A}\left(k_{+}+k_{-}, k\right)\right)=0 \\
& k_{+}^{\mu} \Gamma_{\mu \nu}^{W^{+} Z \phi^{-}}\left(k_{+}, k, k_{-}\right)-M_{W} \Gamma_{\nu}^{\phi^{+} Z \phi^{-}}\left(k_{+}, k, k_{-}\right) \\
& +e \frac{c_{w}}{s_{w}} \Gamma_{\nu}^{W^{+} \phi^{-}}\left(k+k_{+}, k_{-}\right)+\frac{e}{2 s_{w}}\left(\Gamma_{\nu}^{H Z}\left(k_{+}+k_{-}, k\right)+i \Gamma_{\nu}^{\chi Z}\left(k_{+}+k_{-}, k\right)\right)=0 \\
& k_{+}^{\mu} \Gamma_{\mu \nu}^{W^{+} W^{-} H}\left(k_{+}, k_{-}, k\right)-M_{W} \Gamma_{\nu}^{\phi^{+} W^{-} H}\left(k_{+}, k_{-}, k\right) \\
& -\frac{e}{2 s_{w}} \Gamma_{\nu}^{\phi^{+} W^{-}}\left(k+k_{+}, k_{-}\right)+e\left(\Gamma_{\nu}^{A H}\left(k_{+}+k_{-}, k\right)-\frac{c_{w}}{s_{w}} \Gamma_{\nu}^{Z H}\left(k_{+}+k_{-}, k\right)\right)=0
\end{aligned}
$$




$$
\begin{array}{r}
k_{+}^{\mu} \Gamma_{\mu \nu}^{W^{+} W^{-} \chi}\left(k_{+}, k_{-}, k\right)-M_{W} \Gamma_{\nu}^{\phi^{+} W^{-} \chi}\left(k_{+}, k_{-}, k\right) \\
-\frac{i e}{2 s_{w}} \Gamma_{\nu}^{\phi^{+} W^{-}}\left(k+k_{+}, k_{-}\right)+e\left(\Gamma_{\nu}^{A \chi}\left(k_{+}+k_{-}, k\right)-\frac{c_{w}}{s_{w}} \Gamma_{\nu}^{Z \chi}\left(k_{+}+k_{-}, k\right)\right)=0 \\
k_{-}^{\mu} \Gamma_{\mu \nu}^{W^{-} A \phi^{+}}\left(k_{-}, k, k_{+}\right)+M_{W} \Gamma_{\nu}^{\phi^{-}} A \phi^{+}\left(k_{-}, k, k_{+}\right) \\
+e \Gamma_{\nu}^{W^{-} \phi^{+}}\left(k+k_{-}, k_{+}\right)-\frac{e}{2 s_{w}}\left(\Gamma_{\nu}^{H A}\left(k_{+}+k_{-}, k\right)-i \Gamma_{\nu}^{\chi A}\left(k_{+}+k_{-}, k\right)\right)=0 \\
k_{-}^{\mu} \Gamma_{\mu \nu}^{W^{-} Z \phi^{+}}\left(k_{-}, k, k_{+}\right)+M_{W} \Gamma_{\nu}^{\phi^{-} Z \phi^{+}}\left(k_{-}, k, k_{+}\right) \\
-e \frac{c_{w}}{s_{w}} \Gamma_{\nu}^{W^{-} \phi^{+}}\left(k+k_{-}, k_{+}\right)-\frac{e}{2 s_{w}}\left(\Gamma_{\nu}^{H Z}\left(k_{+}+k_{-}, k\right)-i \Gamma_{\nu}^{\chi Z}\left(k_{+}+k_{-}, k\right)\right)=0 \\
+\frac{e}{2 s_{w}} \Gamma_{\nu}^{\phi^{-} W^{+}}\left(k+k_{-}, k_{+}\right)-e\left(\Gamma _ { \nu } ^ { A H } \left(k_{+}+k^{+} H\left(k_{-}, k_{+}, k\right)+M_{W} \Gamma_{\nu}^{\phi^{-} W^{+} H}\left(k_{-}, k_{+}, k\right)\right.\right. \\
\left.k_{w}^{\mu} \Gamma_{\nu}^{Z H}\left(k_{+}+k_{-}, k\right)\right)=0 \\
-\frac{i e}{2 s_{w}} \Gamma_{\nu}^{\phi^{-} W^{+} \chi}\left(k_{-}, k_{+}, k\right)+M_{W} \Gamma_{\nu}^{\phi^{-}} W^{+} \chi\left(k_{-}, k_{+}, k\right)
\end{array}
$$

\section{A.5 Ward identities involving VSS, SSS and SS}

$$
\begin{aligned}
k_{1}^{\mu} \Gamma_{\mu}^{A H H}\left(k_{1}, k_{2}, k_{3}\right)=k_{1}^{\mu} \Gamma_{\mu}^{A H \chi}\left(k_{1}, k_{2}, k_{3}\right)=k_{1}^{\mu} \Gamma_{\mu}^{A \chi \chi}\left(k_{1}, k_{2}, k_{3}\right) & =0 \\
k^{\mu} \Gamma_{\mu}^{A \phi^{+} \phi^{-}}\left(k, k_{+}, k_{-}\right)+e\left(\Gamma^{\phi^{+} \phi^{-}}\left(k+k_{+}, k_{-}\right)-\Gamma^{\phi^{-} \phi^{+}}\left(k+k_{-}, k_{+}\right)\right) & =0 \\
k_{1}^{\mu} \Gamma_{\mu}^{Z H H}\left(k_{1}, k_{2}, k_{3}\right)-i M_{Z} \Gamma^{\chi H H}\left(k_{1}, k_{2}, k_{3}\right)-\frac{i e}{2 c_{w} s_{w}}\left(\Gamma^{\chi H}\left(k_{1}+k_{2}, k_{3}\right)\right. & \left.+\Gamma^{\chi H}\left(k_{1}+k_{3}, k_{2}\right)\right)=0 \\
k_{1}^{\mu} \Gamma_{\mu}^{Z H \chi}\left(k_{1}, k_{2}, k_{3}\right)-i M_{Z} \Gamma^{\chi H \chi}\left(k_{1}, k_{2}, k_{3}\right)-\frac{i e}{2 c_{w} s_{w}}\left(\Gamma^{\chi \chi}\left(k_{1}+k_{2}, k_{3}\right)\right. & \left.-\Gamma^{H H}\left(k_{1}+k_{3}, k_{2}\right)\right)=0 \\
k_{1}^{\mu} \Gamma_{\mu}^{Z \chi \chi}\left(k_{1}, k_{2}, k_{3}\right)-i M_{Z} \Gamma^{\chi \chi \chi}\left(k_{1}, k_{2}, k_{3}\right)+\frac{i e}{2 c_{w} s_{w}}\left(\Gamma^{H \chi}\left(k_{1}+k_{2}, k_{3}\right)\right. & \left.\Gamma^{H \chi}\left(k_{1}+k_{3}, k_{2}\right)\right)=0 \\
-e \frac{c_{w}^{2}-s_{w}^{2}}{2 c_{w} s_{w}}\left(\Gamma^{\phi^{+} \phi^{-}}\left(k+k_{+}, k_{-}\right)-\Gamma^{\phi^{-} \phi^{+}}\left(k+k k_{-}, k_{+}\right)\right) & =0 \\
k_{+}^{\mu} \Gamma_{\mu}^{W^{+} H \phi^{-}}\left(k_{+}, k, k_{-}\right)-M_{W} \Gamma^{\phi^{+} H \phi^{-}}\left(k_{+}, k, k_{-}\right) & \\
+\frac{e}{2 s_{w}}\left(\Gamma^{H H}\left(k_{-}+k_{+}, k\right)+i \Gamma^{\chi H}\left(k_{+}+k_{-}, k\right)\right)-\frac{e}{2 s_{w}} \Gamma^{\phi^{+} \phi^{-}}\left(k_{+}+k, k_{-}\right) & =0 \\
k_{+}^{\mu} \Gamma_{\mu}^{W^{+} \chi \phi^{-}}\left(k_{+}, k, k_{-}\right)-M_{W} \Gamma^{\phi^{+} \chi \phi^{-}}\left(k_{+}, k, k_{-}\right) & \\
+\frac{e}{2 s_{w}}\left(\Gamma^{H \chi}\left(k_{-}+k_{+}, k\right)+i \Gamma^{\chi \chi}\left(k_{+}+k_{-}, k\right)\right)-\frac{i e}{2 s_{w}} \Gamma^{\phi^{+} \phi^{-}}\left(k_{+}+k, k_{-}\right) & =0 \\
\frac{e}{2 s_{w}}\left(\Gamma^{H H}\left(k_{-}+k_{+}, k\right)-i \Gamma^{\chi H}\left(k_{+}+k_{-}, k\right)\right)+\frac{e}{2 s_{w}} \Gamma^{\phi^{-} \phi^{+}}\left(k_{-}+k, k_{+}\right) & =0 \\
k_{-}^{\mu} \Gamma_{\mu}^{W-H \phi^{+}}\left(k_{-}, k, k_{+}\right)+M_{W} \Gamma^{\phi^{-} H \phi^{+}}\left(k_{-}, k, k_{+}\right) &
\end{aligned}
$$




$$
\begin{gathered}
k_{-}^{\mu} \Gamma_{\mu}^{W^{-} \chi \phi^{+}}\left(k_{-}, k, k_{+}\right)+M_{W} \Gamma^{\phi^{-} \chi \phi^{+}}\left(k_{-}, k, k_{+}\right) \\
-\frac{e}{2 s_{w}}\left(\Gamma^{H \chi}\left(k_{-}+k_{+}, k\right)-i \Gamma^{\chi \chi}\left(k_{+}+k_{-}, k\right)\right)-\frac{i e}{2 s_{w}} \Gamma^{\phi^{-} \phi^{+}}\left(k_{-}+k, k_{+}\right)=0
\end{gathered}
$$

\section{A.6 Ward identities involving VVVV, VVVS and VVV}

$$
\begin{aligned}
k_{1,2,3,4}^{\mu} \Gamma_{\mu \nu \kappa \sigma}^{A A A A}\left(k_{1}, k_{2}, k_{3}, k_{4}\right) & =0 \\
k_{1,2,3}^{\mu} \Gamma_{\mu \nu \kappa \sigma}^{A A A Z}\left(k_{1}, k_{2}, k_{3}, k_{4}\right) & =0 \\
k_{1,2}^{\mu} \Gamma_{\mu \nu \kappa \sigma}^{A A Z Z}\left(k_{1}, k_{2}, k_{3}, k_{4}\right) & =0 \\
k_{1}^{\mu} \Gamma_{\mu \nu \kappa \sigma}^{A Z Z Z}\left(k_{1}, k_{2}, k_{3}, k_{4}\right) & =0 \\
k_{1}^{\mu} \Gamma_{\mu \nu \kappa \sigma}^{A A W^{+} W^{-}}\left(k_{1}, k_{2}, k_{+}, k_{-}\right)+e\left[\Gamma_{\nu \kappa \sigma}^{A W^{+} W^{-}}\left(k_{2}, k_{1}+k_{+}, k_{-}\right)\right. & \\
\left.-\Gamma_{\nu \kappa \sigma}^{A W^{+} W^{-}}\left(k_{2}, k_{+}, k_{1}+k_{-}\right)\right] & =0 \\
k_{1}^{\mu} \Gamma_{\mu \nu \kappa \sigma}^{A Z W^{+} W^{-}}\left(k_{1}, k_{2}, k_{+}, k_{-}\right)+e\left[\Gamma_{\kappa \nu \sigma}^{W^{+} Z W^{-}}\left(k_{1}+k_{+}, k_{2}, k_{-}\right)\right. & \\
\left.-\Gamma_{\sigma \nu \kappa}^{W^{-} Z W^{+}}\left(k_{1}+k_{-}, k_{2}, k_{+}\right)\right] & =0 \\
k^{\mu} \Gamma_{\mu \nu \kappa \sigma}^{Z V_{2} V_{3} V_{4}}\left(k_{1}, k_{2}, k_{3}, k_{4}\right)-i M_{Z} \Gamma_{\nu \kappa \sigma}^{\chi V_{2} V_{3} V_{4}}\left(k_{1}, k_{2}, k_{3}, k_{4}\right) & =0,
\end{aligned}
$$

where $k$ here refers to any of the Z momenta and V's stand for A or Z.

$$
\begin{aligned}
k_{+}^{\mu} & \Gamma_{\mu \nu \kappa \sigma}^{W^{+} W^{-} A A}\left(k_{+}, k_{-}, k_{3}, k_{4}\right)+e\left[\Gamma_{\nu \kappa \sigma}^{A A A}\left(k_{+}+k_{-}, k_{3}, k_{4}\right)-\frac{c_{w}}{s_{w}} \Gamma_{\nu \kappa \sigma}^{Z A A}\left(k_{+}+k_{-}, k_{3}, k_{4}\right)\right. \\
& \left.-\Gamma_{\sigma \nu \kappa}^{W^{+} W^{-} A}\left(k_{+}+k_{4}, k_{-}, k_{3}\right)-\Gamma_{\nu \kappa \sigma}^{W^{-} W^{+} A}\left(k_{-}, k_{+}+k_{3}, k_{4}\right)\right] \\
& -M_{W} \Gamma_{\nu \kappa \sigma}^{\phi^{+} W^{-} A A}\left(k_{+}, k_{-}, k_{3}, k_{4}\right)=0 \\
k_{-}^{\mu} \quad & \Gamma_{\mu \nu \kappa \sigma}^{W^{-} W^{+} A A}\left(k_{-}, k_{+}, k_{3}, k_{4}\right)-e\left[\Gamma_{\nu \kappa \sigma}^{A A A}\left(k_{+}+k_{-}, k_{3}, k_{4}\right)-\frac{c_{w}}{s_{w}} \Gamma_{\nu \kappa \sigma}^{Z A A}\left(k_{+}+k_{-}, k_{3}, k_{4}\right)\right. \\
& \left.-\Gamma_{\sigma \nu \kappa}^{W^{-} W^{+} A}\left(k_{-}+k_{4}, k_{+}, k_{3}\right)-\Gamma_{\nu \kappa \sigma}^{W^{+} W^{-} A}\left(k_{+}, k_{-}+k_{3}, k_{4}\right)\right] \\
& +M_{W} \Gamma_{\nu \kappa \sigma}^{\phi^{-} W^{+} A A}\left(k_{-}, k_{+}, k_{3}, k_{4}\right)=0 \\
k_{1}^{\mu} & \Gamma_{\mu \nu \kappa \sigma}^{Z Z W^{+} W^{-}}\left(k_{1}, k_{2}, k_{+}, k_{-}\right)-e \frac{c_{w}}{s_{w}}\left[\Gamma_{\kappa \nu \sigma}^{W^{+} Z W^{-}}\left(k_{1}+k_{+}, k_{2}, k_{-}\right)\right. \\
& \left.-\Gamma_{\sigma \nu \kappa}^{W} Z W^{+}\left(k_{1}+k_{-}, k_{2}, k_{+}\right)\right]-i M_{Z} \Gamma_{\nu \kappa \sigma}^{\chi Z W^{+} W^{-}}\left(k_{1}, k_{2}, k_{+}, k_{-}\right)=0 \\
k_{+}^{\mu} & \Gamma_{\mu \nu \kappa \sigma}^{W^{+} Z Z W^{-}}\left(k_{+}, k_{1}, k_{2}, k_{-}\right)+e\left[\Gamma_{\sigma \nu \kappa}^{A Z Z}\left(k_{+}+k_{-}, k_{1}, k_{2}\right)-\frac{c_{w}}{s_{w}} \Gamma_{\sigma \nu \kappa}^{Z Z Z}\left(k_{+}+k_{-}, k_{1}, k_{2}\right)\right. \\
& \left.+\frac{c_{w}}{s_{w}} \Gamma_{\nu \kappa \sigma}^{W^{+} Z W^{-}}\left(k_{+}+k_{1}, k_{2}, k_{-}\right)+\frac{c_{w}}{s_{w}} \Gamma_{\kappa \nu \sigma}^{W^{+} Z W^{-}}\left(k_{+}+k_{2}, k_{1}, k_{-}\right)\right] \\
& -M_{W} \Gamma_{\nu \kappa \sigma}^{\phi^{+} Z Z W^{-}}\left(k_{+}, k_{1}, k_{2}, k_{-}\right)=0 \\
k_{-}^{\mu} & \Gamma_{\mu \nu \kappa \sigma}^{W-W^{+} Z Z}\left(k_{-}, k_{+}, k_{3}, k_{4}\right)-e\left[\Gamma_{\nu \kappa \sigma}^{A Z Z}\left(k_{+}+k_{-}, k_{3}, k_{4}\right)-\frac{c_{w}}{s_{w}} \Gamma_{\nu \kappa \sigma}^{Z Z Z}\left(k_{+}+k_{-}, k_{3}, k_{4}\right)\right. \\
& \left.+\frac{c_{w}}{s_{w}} \Gamma_{\kappa \nu \sigma}^{W-W^{+} Z}\left(k_{-}+k_{3}, k_{+}, k_{4}\right)+\frac{c_{w}}{s_{w}} \Gamma_{\sigma \nu \kappa}^{W^{-} W^{+} Z}\left(k_{-}+k_{4}, k_{+}, k_{3}\right)\right] \\
& +M_{W} \Gamma_{\nu \kappa \sigma}^{\phi^{-} W^{+} Z Z}\left(k_{-}, k_{+}, k_{3}, k_{4}\right)=0
\end{aligned}
$$




$$
\begin{aligned}
& k_{1}^{\mu} \Gamma_{\mu \nu \kappa \sigma}^{Z A W^{+} W^{-}}\left(k_{1}, k_{2}, k_{+}, k_{-}\right)-e \frac{c_{w}}{s_{w}}\left[\Gamma_{\kappa \nu \sigma}^{W^{+} A W^{-}}\left(k_{1}+k_{+}, k_{2}, k_{-}\right)\right. \\
& \left.-\Gamma_{\sigma \nu \kappa}^{W-A W^{+}}\left(k_{1}+k_{-}, k_{2}, k_{+}\right)\right]-i M_{Z} \Gamma_{\nu \kappa \sigma}^{\chi A W^{+} W^{-}}\left(k_{1}, k_{2}, k_{+}, k_{-}\right)=0 \\
& k_{+}^{\mu} \Gamma_{\mu \nu \kappa \sigma}^{W^{+} W^{-} A Z}\left(k_{+}, k_{-}, k_{3}, k_{4}\right)+e\left[\Gamma_{\nu \kappa \sigma}^{A A Z}\left(k_{+}+k_{-}, k_{3}, k_{4}\right)-\frac{c_{w}}{s_{w}} \Gamma_{\nu \kappa \sigma}^{Z A Z}\left(k_{+}+k_{-}, k_{3}, k_{4}\right)\right. \\
& \left.-\Gamma_{\kappa \nu \sigma}^{W^{+} W^{-} Z}\left(k_{+}+k_{3}, k_{-}, k_{4}\right)+\frac{c_{w}}{s_{w}} \Gamma_{\sigma \nu \kappa}^{W^{+} W^{-} A}\left(k_{+}+k_{4}, k_{-}, k_{3}\right)\right] \\
& -M_{W} \Gamma_{\nu \kappa \sigma}^{\phi^{+} W^{-} A Z}\left(k_{+}, k_{-}, k_{3}, k_{4}\right)=0 \\
& k_{-}^{\mu} \Gamma_{\mu \nu \kappa \sigma}^{W^{-} W^{+} A Z}\left(k_{-}, k_{+}, k_{3}, k_{4}\right)-e\left[\Gamma_{\nu \kappa \sigma}^{A A Z}\left(k_{+}+k_{-}, k_{3}, k_{4}\right)-\frac{c_{w}}{s_{w}} \Gamma_{\nu \kappa \sigma}^{Z A Z}\left(k_{+}+k_{-}, k_{3}, k_{4}\right)\right. \\
& \left.-\Gamma_{\kappa \nu \sigma}^{W^{-} W^{+} Z}\left(k_{-}+k_{3}, k_{+}, k_{4}\right)+\frac{c_{w}}{s_{w}} \Gamma_{\sigma \nu \kappa}^{W^{-} W^{+} A}\left(k_{-}+k_{4}, k_{+}, k_{3}\right)\right] \\
& +M_{W} \Gamma_{\nu \kappa \sigma}^{\phi^{-} W^{+} A Z}\left(k_{-}, k_{+}, k_{3}, k_{4}\right)=0 \\
& k_{1+}^{\mu} \Gamma_{\mu \nu \kappa \sigma}^{W^{+} W^{-} W^{+} W^{-}}\left(k_{1+}, k_{1-}, k_{2+}, k_{2-}\right)+e\left[\Gamma_{\nu \kappa \sigma}^{A W^{+} W^{-}}\left(k_{1+}+k_{1-}, k_{2+}, k_{2-}\right)\right. \\
& -\frac{c_{w}}{s_{w}} \Gamma_{\nu \kappa \sigma}^{Z W^{+} W^{-}}\left(k_{1+}+k_{1-}, k_{2+}, k_{2-}\right)+\Gamma_{\sigma \nu \kappa}^{A W^{-} W^{+}}\left(k_{1+}+k_{2-}, k_{1-}, k_{2+}\right) \\
& \left.-\frac{c_{w}}{s_{w}} \Gamma_{\sigma \nu \kappa}^{Z W^{-} W^{+}}\left(k_{1+}+k_{2-}, k_{1-}, k_{2+}\right)\right]-M_{W} \Gamma_{\nu \kappa \sigma}^{\phi^{+} W^{-} W^{+} W^{-}}\left(k_{1+}, k_{1-}, k_{2+}, k_{2-}\right)=0 \\
& k_{1-}^{\mu} \Gamma_{\mu \nu \kappa \sigma}^{W^{-} W^{+} W^{-} W^{+}}\left(k_{1-}, k_{1+}, k_{2-}, k_{2+}\right)-e\left[\Gamma_{\nu \kappa \sigma}^{A W^{-} W^{+}}\left(k_{1+}+k_{1-}, k_{2-}, k_{2+}\right)\right. \\
& -\frac{c_{w}}{s_{w}} \Gamma_{\nu \kappa \sigma}^{Z W^{-} W^{+}}\left(k_{1+}+k_{1-}, k_{2-}, k_{2+}\right)+\Gamma_{\sigma \nu \kappa}^{A W^{+} W^{-}}\left(k_{2+}+k_{1-}, k_{1+}, k_{2-}\right) \\
& \left.-\frac{c_{w}}{s_{w}} \Gamma_{\sigma \nu \kappa}^{Z W^{+} W^{-}}\left(k_{2+}+k_{1-}, k_{1+}, k_{2-}\right)\right]+M_{W} \Gamma_{\nu \kappa \sigma}^{\phi^{-} W^{+} W^{-} W^{+}}\left(k_{1-}, k_{1+}, k_{2-}, k_{2+}\right)=0
\end{aligned}
$$

\section{A.7 Ward identities involving SSSS, VSSS and SSS}

$$
\begin{aligned}
& k_{1}^{\mu} \Gamma_{\mu}^{Z \chi H H}\left(k_{1}, k_{2}, k_{3}, k_{4}\right)-i M_{Z} \Gamma^{\chi \chi H H}\left(k_{1}, k_{2}, k_{3}, k_{4}\right)-\frac{i e}{2 c_{w} s_{w}}\left[\Gamma^{\chi \chi H}\left(k_{1}+k_{3}, k_{2}, k_{4}\right)\right. \\
&\left.+\Gamma^{\chi \chi H}\left(k_{1}+k_{4}, k_{2}, k_{3}\right)-\Gamma^{H H}\left(k_{1}+k_{2}, k_{3}, k_{4}\right)\right]=0 \\
& k_{1}^{\mu} \quad \Gamma_{\mu}^{Z \chi \chi \chi}\left(k_{1}, k_{2}, k_{3}, k_{4}\right)-i M_{Z} \Gamma^{\chi \chi \chi \chi}\left(k_{1}, k_{2}, k_{3}, k_{4}\right)+\frac{i e}{2 c_{w} s_{w}}\left[\Gamma^{H \chi \chi}\left(k_{1}+k_{2}, k_{3}, k_{4}\right)\right.\left.+\Gamma^{H \chi \chi}\left(k_{1}+k_{3}, k_{2}, k_{4}\right)+\Gamma^{H \chi \chi}\left(k_{1}+k_{4}, k_{2}, k_{3}\right)\right]=0 \\
& k_{1}^{\mu} \quad \Gamma_{\mu}^{Z H \phi^{+} \phi^{-}}\left(k_{1}, k_{2}, k_{+}, k_{-}\right)-i M_{Z} \Gamma^{\chi H \phi^{+} \phi^{-}}\left(k_{1}, k_{2}, k_{+}, k_{-}\right) \\
& \quad-e \frac{c_{w}^{2}-s_{w}^{2}}{2 c_{w} s_{w}}\left[\Gamma^{\phi^{+} H \phi^{-}}\left(k_{1}+k_{+}, k_{2}, k_{-}\right)-\Gamma^{\phi^{-} H \phi^{+}}\left(k_{1}+k_{-}, k_{2}, k_{+}\right)\right] \\
& \quad-\frac{i e}{2 c_{w} s_{w}} \Gamma^{\chi \phi^{+} \phi^{-}}\left(k_{1}+k_{2}, k_{+}, k_{-}\right)=0 \\
& k_{1}^{\mu} \quad \Gamma_{\mu}^{Z \chi \phi^{+} \phi^{-}}\left(k_{1}, k_{2}, k_{+}, k_{-}\right)-i M_{Z} \Gamma^{\chi \chi \phi^{+} \phi^{-}}\left(k_{1}, k_{2}, k_{+}, k_{-}\right) \\
& \quad-e \frac{c_{w}^{2}-s_{w}^{2}}{2 c_{w} s_{w}}\left[\Gamma^{\phi^{+} \chi \phi^{-}}\left(k_{1}+k_{+}, k_{2}, k_{-}\right)-\Gamma^{\phi^{-} \chi \phi^{+}}\left(k_{1}+k_{-}, k_{2}, k_{+}\right)\right] \\
& \quad+\frac{i e}{2 c_{w} s_{w}} \Gamma^{H \phi^{+} \phi^{-}}\left(k_{1}+k_{2}, k_{+}, k_{-}\right)=0
\end{aligned}
$$




$$
\begin{aligned}
k_{+}^{\mu} & \Gamma_{\mu}^{W^{+} \phi^{-} H H}\left(k_{+}, k_{-}, k_{1}, k_{2}\right)-M_{W} \Gamma^{\phi^{+} \phi^{-} H H}\left(k_{+}, k_{-}, k_{1}, k_{2}\right) \\
& +\frac{e}{2 s_{w}}\left[\Gamma^{H H H}\left(k_{+}+k_{-}, k_{1}, k_{2}\right)+i \Gamma^{\chi H H}\left(k_{+}+k_{-}, k_{1}, k_{2}\right)\right. \\
& \left.-\Gamma^{\phi^{+} \phi^{-} H}\left(k_{1}+k_{+}, k_{-}, k_{2}\right)-\Gamma^{\phi^{+} \phi^{-} H}\left(k_{2}+k_{+}, k_{-}, k_{1}\right)\right]=0 \\
k_{+}^{\mu} & \Gamma_{\mu}^{W^{+} \phi^{-} H \chi}\left(k_{+}, k_{-}, k_{1}, k_{2}\right)-M_{W} \Gamma^{\phi^{+} \phi^{-} H \chi}\left(k_{+}, k_{-}, k_{1}, k_{2}\right)+\frac{e}{2 s_{w}}\left[\Gamma^{H H \chi}\left(k_{+}+k_{-}, k_{1}, k_{2}\right)\right. \\
& \left.+i \Gamma^{\chi H \chi}\left(k_{+}+k_{-}, k_{1}, k_{2}\right)-\Gamma^{\phi^{+} \phi^{-} \chi}\left(k_{1}+k_{+}, k_{-}, k_{2}\right)-i \Gamma^{\phi^{+} \phi^{-} H}\left(k_{2}+k_{+}, k_{-}, k_{1}\right)\right]=0
\end{aligned}
$$

$$
\begin{aligned}
k_{+}^{\mu} & \Gamma_{\mu}^{W^{+} \phi^{-} \chi \chi}\left(k_{+}, k_{-}, k_{1}, k_{2}\right)-M_{W} \Gamma^{\phi^{+} \phi^{-}} \chi \chi\left(k_{+}, k_{-}, k_{1}, k_{2}\right) \\
& +\frac{e}{2 s_{w}}\left[\Gamma^{H \chi \chi}\left(k_{+}+k_{-}, k_{1}, k_{2}\right)+i \Gamma^{\chi \chi \chi}\left(k_{+}+k_{-}, k_{1}, k_{2}\right)\right. \\
& \left.-i \Gamma^{\phi^{+} \phi^{-} \chi}\left(k_{1}+k_{+}, k_{-}, k_{2}\right)-i \Gamma^{\phi^{+} \phi^{-} \chi}\left(k_{2}+k_{+}, k_{-}, k_{1}\right)\right]=0 \\
k_{1+}^{\mu} & \Gamma_{\mu}^{W^{+} \phi^{-} \phi^{+} \phi^{-}}\left(k_{1+}, k_{1-}, k_{2+}, k_{2-}\right)-M_{W} \Gamma^{\phi^{+} \phi^{-} \phi^{+} \phi^{-}}\left(k_{1+}, k_{1-}, k_{2+}, k_{2-}\right)
\end{aligned}
$$$$
+\frac{e}{2 s_{w}}\left[\Gamma^{H \phi^{+} \phi^{-}}\left(k_{1+}+k_{1-}, k_{2+}, k_{2-}\right)+i \Gamma^{\chi \phi^{+} \phi^{-}}\left(k_{1+}+k_{1-}, k_{2+}, k_{2-}\right)\right.
$$$$
\left.+\Gamma^{H \phi^{-} \phi^{+}}\left(k_{1+}+k_{2-}, k_{1-}, k_{2+}\right)+i \Gamma^{\chi \phi^{-} \phi^{+}}\left(k_{1+}+k_{2-}, k_{1-}, k_{2+}\right)\right]=0
$$$$
k_{-}^{\mu} \Gamma_{\mu}^{W^{-} \phi^{+} H H}\left(k_{-}, k_{+}, k_{1}, k_{2}\right)+M_{W} \Gamma^{\phi^{-} \phi^{+} H H}\left(k_{-}, k_{+}, k_{1}, k_{2}\right)
$$$$
-\frac{e}{2 s_{w}}\left[\Gamma^{H H H}\left(k_{+}+k_{-}, k_{1}, k_{2}\right)-i \Gamma^{\chi H H}\left(k_{+}+k_{-}, k_{1}, k_{2}\right)\right.
$$$$
\left.-\Gamma^{\phi^{-} \phi^{+} H}\left(k_{1}+k_{-}, k_{+}, k_{2}\right)-\Gamma^{\phi^{-} \phi^{+} H}\left(k_{2}+k_{-}, k_{+}, k_{1}\right)\right]=0
$$$$
k_{-}^{\mu} \Gamma_{\mu}^{W^{-} \phi^{+} H \chi}\left(k_{-}, k_{+}, k_{1}, k_{2}\right)+M_{W} \Gamma^{\phi^{-} \phi^{+} H \chi}\left(k_{-}, k_{+}, k_{1}, k_{2}\right)
$$$$
-\frac{e}{2 s_{w}}\left[\Gamma^{H H \chi}\left(k_{+}+k_{-}, k_{1}, k_{2}\right)-i \Gamma^{\chi H \chi}\left(k_{+}+k_{-}, k_{1}, k_{2}\right)\right.
$$$$
\left.-\Gamma^{\phi^{-} \phi^{+} \chi}\left(k_{1}+k_{-}, k_{+}, k_{2}\right)+i \Gamma^{\phi^{-} \phi^{+} H}\left(k_{2}+k_{-}, k_{+}, k_{1}\right)\right]=0
$$$$
k_{-}^{\mu} \Gamma_{\mu}^{W^{-} \phi^{+} \chi \chi}\left(k_{-}, k_{+}, k_{1}, k_{2}\right)+M_{W} \Gamma^{\phi^{-} \phi^{+}} \chi \chi\left(k_{-}, k_{+}, k_{1}, k_{2}\right)
$$$$
-\frac{e}{2 s_{w}}\left[\Gamma^{H \chi \chi}\left(k_{+}+k_{-}, k_{1}, k_{2}\right)-i \Gamma^{\chi \chi \chi}\left(k_{+}+k_{-}, k_{1}, k_{2}\right)\right.
$$$$
\left.+i \Gamma^{\phi^{-} \phi^{+} \chi}\left(k_{1}+k_{-}, k_{+}, k_{2}\right)+i \Gamma^{\phi^{-} \phi^{+} \chi}\left(k_{2}+k_{-}, k_{+}, k_{1}\right)\right]=0
$$$$
k_{1-}^{\mu} \Gamma_{\mu}^{W^{-} \phi^{+} \phi^{-} \phi^{+}}\left(k_{1-}, k_{1+}, k_{2-}, k_{2+}\right)+M_{W} \Gamma^{\phi^{-} \phi^{+} \phi^{-} \phi^{+}}\left(k_{1-}, k_{1+}, k_{2-}, k_{2+}\right)
$$$$
-\frac{e}{2 s_{w}}\left[\Gamma^{H \phi^{-} \phi^{+}}\left(k_{1+}+k_{1-}, k_{2-}, k_{2+}\right)-i \Gamma^{\chi \phi^{-} \phi^{+}}\left(k_{1+}+k_{1-}, k_{2-}, k_{2+}\right)\right.
$$$$
\left.+\Gamma^{H \phi^{+} \phi^{-}}\left(k_{2+}+k_{1-}, k_{1+}, k_{2-}\right)-i \Gamma^{\chi \phi^{+} \phi^{-}}\left(k_{2+}+k_{1-}, k_{1+}, k_{2-}\right)\right]=0
$$

Open Access. This article is distributed under the terms of the Creative Commons Attribution Noncommercial License which permits any noncommercial use, distribution, and reproduction in any medium, provided the original author(s) and source are credited. 


\section{References}

[1] G. Bevilacqua, M. Czakon, C.G. Papadopoulos, R. Pittau and M. Worek, Assault on the NLO wishlist: $p p \rightarrow t t b b$, JHEP 09 (2009) 109 [arXiv:0907.4723] [SPIRES].

[2] R.K. Ellis, K. Melnikov and G. Zanderighi, Generalized unitarity at work: first NLO QCD results for hadronic $W^{+}$3jet production, JHEP 04 (2009) 077 [arXiv:0901.4101] [SPIRES].

[3] C.F. Berger et al., Precise predictions for $W+3$ jet production at Hadron colliders, Phys. Rev. Lett. 102 (2009) 222001 [arXiv:0902.2760] [SPIRES].

[4] T. Binoth, G. Ossola, C.G. Papadopoulos and R. Pittau, NLO QCD corrections to tri-boson production, JHEP 06 (2008) 082 [arXiv:0804.0350] [SPIRES].

[5] P. Mastrolia, G. Ossola, C.G. Papadopoulos and R. Pittau, Optimizing the reduction of one-loop amplitudes, JHEP 06 (2008) 030 [arXiv:0803.3964] [SPIRES].

[6] G. Ossola, C.G. Papadopoulos and R. Pittau, Reducing full one-loop amplitudes to scalar integrals at the integrand level, Nucl. Phys. B 763 (2007) 147 [hep-ph/0609007] [SPIRES].

[7] R. Britto, F. Cachazo and B. Feng, Generalized unitarity and one-loop amplitudes in $N=4$ super-Yang-Mills, Nucl. Phys. B 725 (2005) 275 [hep-th/0412103] [SPIRES].

[8] Z. Bern, L.J. Dixon and D.A. Kosower, One loop corrections to five gluon amplitudes, Phys. Rev. Lett. 70 (1993) 2677 [hep-ph/9302280] [SPIRES].

[9] Z. Bern, L.J. Dixon and D.A. Kosower, One loop corrections to two quark three gluon amplitudes, Nucl. Phys. B 437 (1995) 259 [hep-ph/9409393] [SPIRES].

[10] Z. Bern, L.J. Dixon and D.A. Kosower, One-loop amplitudes for $e^{+} e^{-}$to four partons, Nucl. Phys. B 513 (1998) 3 [hep-ph/9708239] [SPIRES].

[11] A. van Hameren, C.G. Papadopoulos and R. Pittau, Automated one-loop calculations: a proof of concept, JHEP 09 (2009) 106 [arXiv:0903.4665] [SPIRES].

[12] F. Caravaglios and M. Moretti, An algorithm to compute Born scattering amplitudes without Feynman graphs, Phys. Lett. B 358 (1995) 332 [hep-ph/9507237] [SPIRES].

[13] C.G. Papadopoulos and M. Worek, Multi-parton cross sections at hadron colliders, Eur. Phys. J. C 50 (2007) 843 [hep-ph/0512150] [SPIRES].

[14] M.L. Mangano, M. Moretti, F. Piccinini, R. Pittau and A.D. Polosa, ALPGEN, a generator for hard multiparton processes in hadronic collisions, JHEP 07 (2003) 001 [hep-ph/0206293] [SPIRES].

[15] A. Cafarella, C.G. Papadopoulos and M. Worek, Helac-Phegas: a generator for all parton level processes, Comput. Phys. Commun. 180 (2009) 1941 [arXiv:0710.2427] [SPIRES].

[16] C. Anastasiou, R. Britto, B. Feng, Z. Kunszt and P. Mastrolia, D-dimensional unitarity cut method, Phys. Lett. B 645 (2007) 213 [hep-ph/0609191] [SPIRES].

[17] R.K. Ellis, W.T. Giele, Z. Kunszt and K. Melnikov, Masses, fermions and generalized D-dimensional unitarity, Nucl. Phys. B 822 (2009) 270 [arXiv:0806.3467] [SPIRES].

[18] W.T. Giele, Z. Kunszt and K. Melnikov, Full one-loop amplitudes from tree amplitudes, JHEP 04 (2008) 049 [arXiv:0801.2237] [SPIRES].

[19] Z. Bern, L.J. Dixon and D.A. Kosower, On-shell recurrence relations for one-loop QCD amplitudes, Phys. Rev. D 71 (2005) 105013 [hep-th/0501240] [SPIRES]. 
[20] Z. Bern, L.J. Dixon and D.A. Kosower, The last of the finite loop amplitudes in QCD, Phys. Rev. D 72 (2005) 125003 [hep-ph/0505055] [SPIRES].

[21] Z. Bern, L.J. Dixon and D.A. Kosower, Bootstrapping multi-parton loop amplitudes in QCD, Phys. Rev. D 73 (2006) 065013 [hep-ph/0507005] [SPIRES].

[22] C.F. Berger, Z. Bern, L.J. Dixon, D. Forde and D.A. Kosower, Bootstrapping one-loop QCD amplitudes with general helicities, Phys. Rev. D 74 (2006) 036009 [hep-ph/0604195] [SPIRES].

[23] A. van Hameren, Multi-gluon one-loop amplitudes using tensor integrals, JHEP 07 (2009) 088 [arXiv:0905.1005] [SPIRES].

[24] G. Ossola, C.G. Papadopoulos and R. Pittau, On the rational terms of the one-loop amplitudes, JHEP 05 (2008) 004 [arXiv:0802.1876] [SPIRES].

[25] P. Draggiotis, M.V. Garzelli, C.G. Papadopoulos and R. Pittau, Feynman rules for the rational part of the QCD 1-loop amplitudes, JHEP 04 (2009) 072 [arXiv:0903.0356] [SPIRES].

[26] Z. Kunszt, A. Signer and Z. Trócsányi, One loop helicity amplitudes for all $2 \rightarrow 2$ processes in QCD and $N=1$ supersymmetric Yang-Mills theory, Nucl. Phys. B 411 (1994) 397 [hep-ph/9305239] [SPIRES].

[27] S. Catani, M.H. Seymour and Z. Trócsányi, Regularization scheme independence and unitarity in QCD cross sections, Phys. Rev. D 55 (1997) 6819 [hep-ph/9610553] [SPIRES].

[28] Z. Bern, A. De Freitas, L.J. Dixon and H.L. Wong, Supersymmetric regularization, two-loop QCD amplitudes and coupling shifts, Phys. Rev. D 66 (2002) 085002 [hep-ph/0202271] [SPIRES].

[29] G. Ossola, C.G. Papadopoulos and R. Pittau, Numerical evaluation of six-photon amplitudes, JHEP 07 (2007) 085 [arXiv: 0704.1271] [SPIRES].

[30] G. Ossola, C.G. Papadopoulos and R. Pittau, CutTools: a program implementing the OPP reduction method to compute one-loop amplitudes, JHEP 03 (2008) 042 [arXiv:0711.3596] [SPIRES].

[31] T. Binoth, J.P. Guillet and G. Heinrich, Algebraic evaluation of rational polynomials in one-loop amplitudes, JHEP 02 (2007) 013 [hep-ph/0609054] [SPIRES].

[32] A. Bredenstein, A. Denner, S. Dittmaier and S. Pozzorini, NLO QCD corrections to top anti-top bottom anti-bottom production at the LHC: 1. quark-antiquark annihilation, JHEP 08 (2008) 108 [arXiv:0807.1248] [SPIRES].

[33] J.A.M. Vermaseren, New features of FORM, math-ph/0010025 [SPIRES].

[34] J.A.M. Vermaseren, The FORM project, Nucl. Phys. Proc. Suppl. 183 (2008) 19 [arXiv:0806.4080] [SPIRES].

[35] A. Denner, Techniques for calculation of electroweak radiative corrections at the one loop level and results for W physics at LEP-200, Fortschr. Phys. 41 (1993) 307 [arXiv: 0709.1075] [SPIRES].

[36] T. Hahn, Generating Feynman diagrams and amplitudes with FeynArts 3, Comput. Phys. Commun. 140 (2001) 418 [hep-ph/0012260] [SPIRES].

[37] A. Denner, G. Weiglein and S. Dittmaier, Application of the background field method to the electroweak standard model, Nucl. Phys. B 440 (1995) 95 [hep-ph/9410338] [SPIRES]. 
[38] G. Passarino and M.J.G. Veltman, One loop corrections for $e^{+} e^{-}$annihilation into $\mu^{+} \mu^{-}$in the Weinberg model, Nucl. Phys. B 160 (1979) 151 [SPIRES].

[39] A. Denner, G. Weiglein and S. Dittmaier, Gauge invariance of green functions: Background field method versus pinch technique, Phys. Lett. B 333 (1994) 420 [hep-ph/9406204] [SPIRES].

[40] A. Denner, S. Dittmaier and G. Weiglein, Green functions from a gauge-invariant effective action for the electroweak standard model, Nucl. Phys. Proc. Suppl. 37B (1994) 87 [hep-ph/9406400] [SPIRES].

[41] A. Denner, S. Dittmaier and G. Weiglein, Gauge invariance, gauge parameter independence and properties of Green functions, hep-ph/9505271 [SPIRES].

[42] A. Denner, S. Dittmaier and G. Weiglein, The background-field formulation of the electroweak standard model, Acta Phys. Polon. B 27 (1996) 3645 [hep-ph/9609422] [SPIRES].

[43] D.Y. Bardin and G. Passarino, The standard model in the making: precision study of the electroweak interactions, Clarendon, Oxford, U.K. (1999) pg. 685 [SPIRES]. 